\title{
Madecassic acid, the contributor to the anti-colitis effect of madecassoside, enhances the shift of Th17 toward Treg cells via the PPARy/AMPK/ACC1 pathway
}

\author{
Xiaotian $\mathrm{Xu}^{1,2}$, Yuhui Wang ${ }^{1,2}$, Zhifeng Wei ${ }^{1}$, Wenhui Wei ${ }^{1}$, Peng Zhao ${ }^{1}$, Bei Tong ${ }^{1}$, Yufeng $\mathrm{Xia}^{\star, 1}$ and Yue Dai ${ }^{\star, 1}$
}

The imbalance between Th17 and Treg cells substantially contributes to the intestinal immune disturbance and subsequent tissue injury in ulcerative colitis. The triterpenoid-rich fraction of Centella asiatica was able to ameliorate dextran sulfate sodium-induced colitis in mice. Here we explored its active ingredient and underlying mechanism with a focus on restoring the Th17/Treg balance. The four main triterpenoids occurring in $C$. asiatica were shown to attenuate colitis in mice by oral administration. The most effective ingredient madecassoside lost anti-colitis effect when applied topically in the colon, and madecassic acid was recognized to be the active form of madecassoside. Oral administration of madecassic acid decreased the percentage of Th17 cells and downregulated the expression of RORyt, IL-17A, IL-17F, IL-21 and IL-22 and increased the percentage of Treg cells and the expression of Foxp3 and IL-10 in the colons of mice with colitis, but it did not affect Th1 and Th2 cells. Under Th17-polarizing conditions, madecassic acid downregulated ACC1 expression and enhanced the shift of Th17 cells toward Treg cells, but it did not affect the differentiation of Treg cells under Treg-polarizing conditions. Both compound C and AMPK siRNA inhibited the madecassic acid-mediated downregulation of ACC1 expression and shift of Th17 cells to Treg cells under Th17-polarizing conditions. GW9662, T0070907 and PPAR $\gamma$ siRNA blocked the effect of madecassic acid on AMPK activation, ACC1 expression and shift of Th17 cells to Treg cells. Furthermore, madecassic acid was identified as a PPAR $\gamma$ agonist, as it promoted PPAR $\gamma$ transactivation. The correlation between activation of PPAR $\gamma$ and AMPK, downregulation of ACC1 expression, restoration of Th17/Treg balance and attenuation of colitis by madecassic acid was validated in mice with DSS-induced colitis. In conclusion, madecassic acid was the active form of madecassoside in ameliorating colitis by restoring the Th17/Treg balance via regulating the PPAR $\gamma / A M P K / A C C 1$ pathway.

Cell Death and Disease (2017) 8, e2723; doi:10.1038/cddis.2017.150; published online 30 March 2017

Ulcerative colitis (UC) is a debilitating syndrome characterized by colonic mucosal ulceration, abdominal pain and intestinal barrier dysfunction. ${ }^{1,2}$ Immune system dysfunction, particularly an imbalance of Th17 cells and Treg cells, contributes substantially to the occurrence and development of UC..$^{3-5}$ An excess of Th17 cells and insufficient Treg cells result in persistent immune dysfunction and sustained intestinal inflammation. ${ }^{6-8}$ Therefore, restoring the balance of Th17/ Treg cells may be a practical therapeutic strategy for treating UC.

Centella asiatica (L.) Urban, a perennial herbaceous plant with pleiotropic bioactivities, mainly consists of pentacyclic triterpenes, including the glycosides madecassoside and asiaticoside as well as their corresponding aglycones madecassic acid and asiatic acid. ${ }^{9-11}$ Our previous studies demonstrated that the triterpenoid-rich fraction of this herb could ameliorate dextran sulfate sodium (DSS)-induced colitis in mice (unpublished data). Madecassoside, the most abundant triterpene in this herb, was shown to regulate the balance of Th17/Treg cells in a collagen-induced arthritis in rats. ${ }^{12}$ Whether it functions as the primary active ingredient of C. asiatica in ameliorating colitis by restoring the Th17/Treg balance remains to be determined.

The balance of Th17/Treg cells can be restored by reducing the generation of Th17 cells, promoting the development of Treg cells and enhancing the phenotypic shift between Th17 and Treg cells. ${ }^{13,14}$ Accumulative evidence suggests that nuclear receptors, especially peroxisome proliferatoractivated receptor $\gamma$ (PPAR $y)$, has a vital role in regulating Th17/Treg balance. ${ }^{15-17}$ The PPAR $y$ agonists inhibit Th17 cell differentiation in lung myeloid dendritic cells and promote Treg cell differentiation in the white adipose tissue of mice. ${ }^{18-21}$ Meanwhile, various pentacyclic triterpenes were reported to activate PPAR ${ }^{22,23}$ These findings suggested that the triterpenes in C. asiatica might restore the Th17/Treg balance through the PPARy pathway.

The present study aimed to identify the primary active ingredient of $C$. asiatica and explore its underlying mechanisms for anti-UC potential with an emphasis on the Th17/Treg balance.

${ }^{1}$ Department of Pharmacology of Chinese Materia Medica, China Pharmaceutical University, 24 Tong Jia Xiang, Nanjing 210009, China

*Corresponding author: Y Dai or Y Xia, Department of Pharmacology of Chinese Materia Medica, China Pharmaceutical University, 24 Tong Jia Xiang, Nanjing 210009 , China. Tel: +86 25 83271400; Fax: +86 25 85301528; E-mail: yuedaicpu@ @otmail.com or yfxiacpu@ 126.com

${ }^{2}$ These authors contributed equally to this work.

Received 19.11.16; revised 20.2.17; accepted 20.2.17; Edited by H-U Simon 


\section{Results}

Madecassoside, the main ingredient of $C$. asiatica, attenuated DSS-induced colitis in mice through its aglycone madecassic acid. Drinking DSS induces severe colitis in mice. ${ }^{24}$ To clarify the active ingredients for the anticolitis effect of $C$. asiatica, four major pentacyclic triterpenes isolated from the plant (madecassoside, asiaticoside, madecassic acid and asiatic acid, Figure 1a) and cyclosporin A were orally administered to DSS-treated mice for 10 days. Madecassoside $(50 \mathrm{mg} / \mathrm{kg})$ and madecassic acid $(25 \mathrm{mg} / \mathrm{kg})$ were shown to be more effective than asiaticoside $(50 \mathrm{mg} / \mathrm{kg}$ ) and asiatic acid (25 mg/kg), respectively, as evidenced by decreasing disease activity index (DAI) scores (Figure 1b), reducing colon shortening (Figure 1c), lowering myeloperoxidase (MPO) activity (Figure 1d) and attenuating pathological injuries of colonic tissues (Figure 1e and Supplementary Figure S1). It appeared that pentacyclic triterpenes with a hydroxyl group were superior to those without a hydroxyl group in ameliorating colitis in mice.

Madecassoside will rapidly metabolize into its aglycone madecassic acid in the small intestine after oral administration. It was necessary to identify the efficient form of madecassoside for attenuating colitis. Our data showed that intra-rectal administration of madecassic acid $(25 \mathrm{mg} / \mathrm{kg})$ effectively ameliorated colitis in mice, as confirmed by reducing DAI scores (Figure 1f), protecting against colon shortening (Figure 1g), decreasing MPO activity (Figure 1h) and attenuating pathological lesions (Figure $1 \mathrm{i}$ and Supplementary Figure S1). In contrast, madecassoside $(50 \mathrm{mg} / \mathrm{kg}$ ) per rectum failed to protect against pathological injury in the colons of mice. These findings revealed that the primary active ingredient madecassoside acted through the intestinal metabolite madecassic acid in ameliorating colitis in mice.

Madecassic acid restored the Th17/Treg balance in mice with DSS-induced colitis. Madecassic acid (12.5, $25 \mathrm{mg} / \mathrm{kg}$ ) conferred protection against DSS-treated colitis in mice (Figures 2a-d and Supplementary Figure S1). DSS-treated mice showed higher percentages of $\mathrm{CD} 4^{+} \mathrm{IFN}-\gamma^{+} \mathrm{T}$ cells and $\mathrm{CD} 4^{+} \mathrm{IL}-17^{+} \mathrm{T}$ cells but not CD4 ${ }^{+} \mathrm{IL}-4^{+} \mathrm{T}$ cells or $\mathrm{CD} 4^{+} \mathrm{CD} 25^{+}$ Foxp $3^{+} \mathrm{T}$ cells in the colonic lamina propria. Madecassic acid considerably decreased the percentage of $\mathrm{CD} 4^{+} \mathrm{IL}-17^{+} \mathrm{T}$ cells and increased the percentage of $\mathrm{CD}^{+}{ }^{+} \mathrm{CD} 25^{+} \mathrm{Foxp} 3^{+} \mathrm{T}$ cells without marked effects on the percentages of $\mathrm{CD} 4^{+} \mathrm{IFN}-\gamma^{+}$and $\mathrm{CD} 4^{+} \mathrm{IL}-4^{+} \mathrm{T}$ cells (Figure $2 \mathrm{e}$ and Supplementary Figure S1). Noteworthy, the ratio of Th17/Treg cells was 0.33 in the normal group, and it climbed to 1.15 in the model group (Figure $2 \mathrm{e}$ and Supplementary Figure S1). Madecassic acid (12.5, $25 \mathrm{mg} / \mathrm{kg}$ ) decreased the ratio of Th17/Treg cells to 0.46 and 0.34 , respectively. These data suggested that madecassic acid could restore the balance of Th17/Treg cells in the colons. Consistently, madecassic acid (25 mg/ $/ \mathrm{kg})$ reduced the expression of Th17 cell transcription factor RORyt, it $(12.5,25 \mathrm{mg} / \mathrm{kg})$ enhanced the expression of Treg cell transcription factor Foxp3, but it did not affect the expression of T-bet and GATA-3 (Figures 2f, $g$ and Supplementary Figure S1). Additionally, madecassic acid $(12.5,25 \mathrm{mg} / \mathrm{kg})$ downregulated the expression of Th17 cell-specific cytokines, while upregulated the expression of Treg cell-specific cytokine IL-10 in the colons (Figures $2 \mathrm{~h}$ and i). Taken together, madecassic acid alleviated DSS-induced colitis in mice by restoring the balance of Th17/ Treg cells in the colon.

Madecassic acid restored the Th17/Treg balance by enhancing the shift of Th17 toward Treg cells. To recognize how madecassic acid restores the Th17/Treg balance, naive mouse $\mathrm{CD}^{+} \mathrm{T}$ cells were cultured under Th17- or Treg-polarizing conditions. ${ }^{25,26}$ Madecassic acid $(3,10 \mu \mathrm{M})$ decreased the percentage of CD4 ${ }^{+} \mathrm{IL}-17^{+} \mathrm{T}$ cells but increased the percentage of $\mathrm{CD}^{+}{ }^{+} \mathrm{Foxp}^{+} \mathrm{T}$ cells under Th17-polarizing conditions (Figure $3 \mathrm{a}$ and Supplementary Figure S1). But it showed very weak effect on the percentages of CD4 ${ }^{+} \mathrm{IL}-17^{+}$ $\mathrm{T}$ cells and $\mathrm{CD}^{+}{ }^{+} \mathrm{Foxp}^{+} \mathrm{T}$ cells under Treg-polarizing conditions (Figure $3 \mathrm{~b}$ and Supplementary Figure S1). Consistently, madecassic acid $(3,10 \mu \mathrm{M})$ downregulated the expression of Th17 cell-specific cytokines and upregulated the expression of IL-10 under Th17-polarizing conditions (Figure 3c), but it did not affect the expression of IL-10 under Treg-polarizing conditions (Supplementary Figure S2a). Consequently, we postulated that madecassic acid might induce the shift of Th17 toward Treg cells under Th17polarizing conditions instead of directly enhancing Treg differentiation.

To recognize whether the madecassic acid-induced shift of Th17 toward Treg cells was achieved by inhibiting conventional Th17 cell differentiation pathways, we investigated the effect of madecassic acid on Th17 and Treg transcription factors and differentiation-associated signal transducers under Th17-polarizing conditions and in DSS-induced mice. Madecassic acid $(3 \mu \mathrm{M})$ showed weak inhibition on the expression of RORyt but obviously enriched the expression of Foxp3 under Th17-polarizing conditions (Figures 3d-f and Supplementary Figure S1), implying that madecassic acid promoted the shift of Th17 toward Treg cells by inducing Foxp3 expression instead of reducing RORyt expression. In contrast, the expression of Foxp3 was not affected by madecassic acid under Treg-polarizing conditions (Supplementary Figure S2b). Furthermore, madecassic acid only weakly inhibited the phosphorylation of STAT3, JAK2, AKT and translocation of STAT3 under Th17-polarizing conditions (Figure $3 e$ and Supplementary Figure S1), implying that madecassic acidinduced shift of Th17 toward Treg cells might not be through the inhibition of conventional Th17 cell differentiation pathways. Similarly, madecassic acid $(12.5,25 \mathrm{mg} / \mathrm{kg}$ ) showed a little inhibition on the phosphorylation of STAT3, JAK2 and AKT in the colons of DSS-induced mice (Supplementary Figure S2d). The phosphorylation of Treg cell activationassociated signal transducers STAT5 and Smad3 were not affected by madecassic acid under Treg-polarizing conditions and in DSS-induced mice (Supplementary Figures S2c and d). These findings indicated that madecassic acid might not affect the activation of STAT3, JAK2 and AKT in the shift of Th17 toward Treg cells.

Madecassic acid facilitated the shift of Th17 toward Treg cells by suppressing the acetyl CoA carboxylase (ACC1) expression. ACC1, the rate-limiting enzyme in fatty acid synthesis that catalyzes the carboxylation of acetyl-CoA to 
a

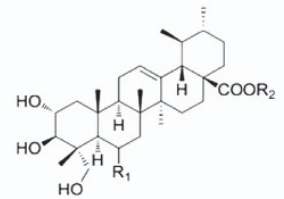

(1) Madecassoside $\mathrm{R}_{1}=\mathrm{OHR}_{2}=-\beta-D-g / c$ $(6 \rightarrow 1)-\beta-D$-glc- $(4 \rightarrow 1)$-a- $L-r h a$

(2) Asiaticoside $\quad \mathrm{R}_{1}=\mathrm{H} \quad \mathrm{R}_{2}=-\beta-D-g / c$ $(6 \rightarrow 1)-\beta-D-g / c-(4,1)-a-L-r h a$

(3) Madecassic acid $\mathrm{R}_{1}=\mathrm{OHR}_{2}=\mathrm{H}$

$\begin{array}{ll}\text { (4) Asiatic acid } \quad R_{1}=H \quad R_{2}=H & \end{array}$

C

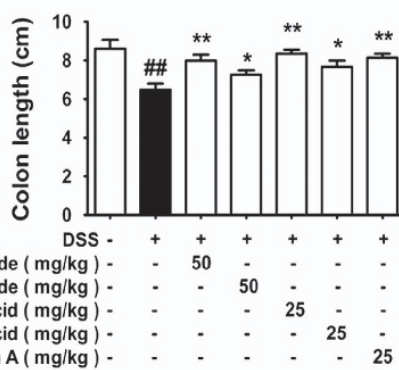

d

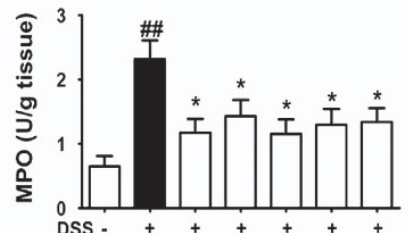

Madecassoside ( $\mathrm{mg} / \mathrm{kg}$ ) Asiaticoside $(\mathrm{mg} / \mathrm{kg}$ ) Madecassic acid ( $\mathrm{mg} / \mathrm{kg}$ )

Asiatic acid ( $\mathrm{mg} / \mathrm{kg}$ ) Cyclosporin A ( $\mathrm{mg} / \mathrm{kg}$ )

f

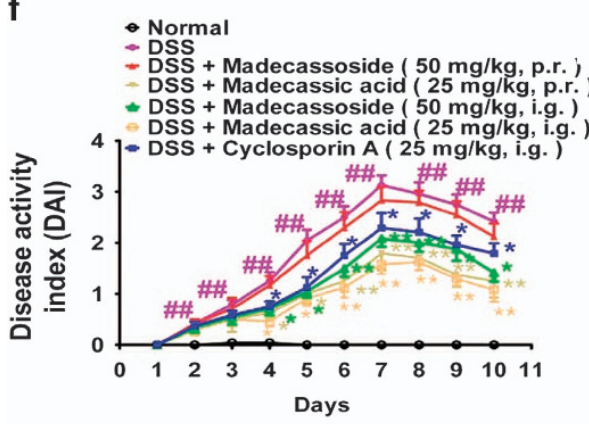

h

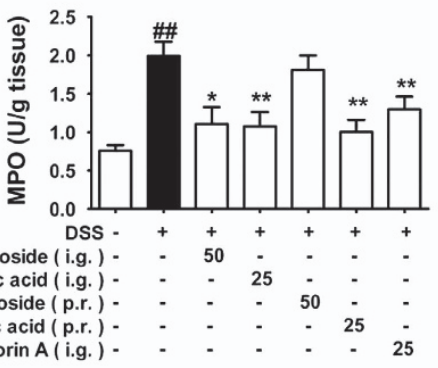

b

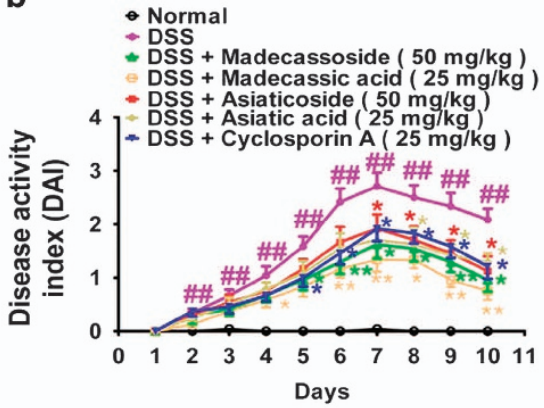

e
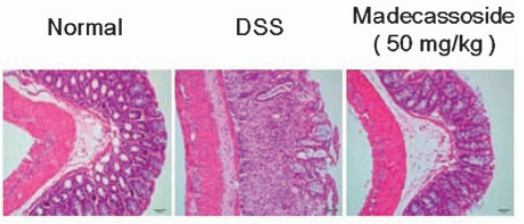

Asiaticoside Madecassic acid Asiatic acid Cyclosporin A

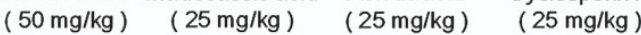

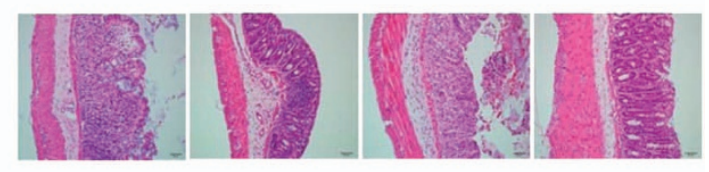

g

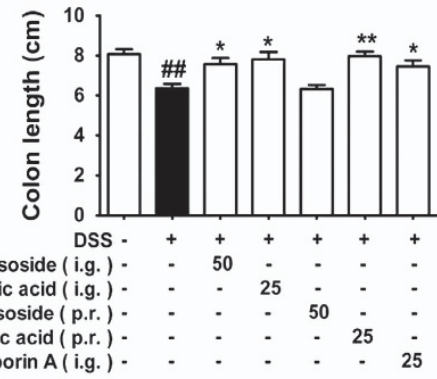

i

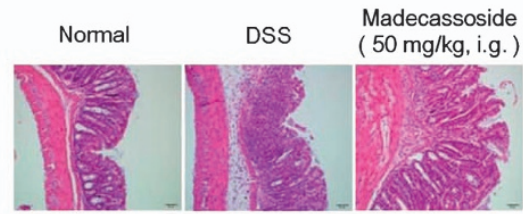

Madecassic acid Madecassoside Madecassic acid Cyclosporin A ( $25 \mathrm{mg} / \mathrm{kg}$, i.g. ) ( $50 \mathrm{mg} / \mathrm{kg}$, p.r. ) ( $25 \mathrm{mg} / \mathrm{kg}$, p.r. ) ( $25 \mathrm{mg} / \mathrm{kg}$, i.g. )

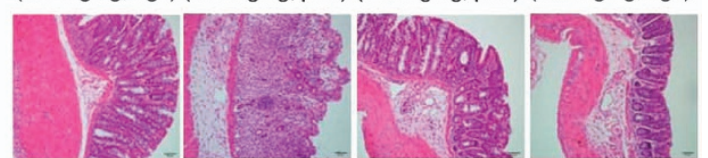

Figure 1 Madecassic acid was the active form of madecassoside in ameliorating DSS-induced colitis in mice. Mice were fed with $2.5 \%$ DSS for 7 days and then given normal water for an additional 3 days. Madecassoside (50 mg/kg), asiaticoside (50 mg/kg), madecassic acid (25 mg/kg), asiatic acid (25 mg/kg) and cyclosporin A (25 mg/kg) were orally administered for consecutive 10 days. (a) Chemical structures of madecassoside, asiaticoside, madecassic acid and asiatic acid. (b) DAl. (c) The colon length of each group at day 10. (d) The activity of MPO in the colons. (e) The epithelial damage, inflammatory cell infiltration and crypt lesions were evaluated by H\&E staining. (f) Mice were rectally administered test compounds for 10 days. DAI. (g) Colon length. (h) MPO activity. (i) H\&E staining. The data were expressed as means \pm S.E.M., $n=5-8$. ${ }^{\#} P<0.01$ versus normal group; ${ }^{*} P<0.05,{ }^{* \star} P<0.01$ versus DSS group 


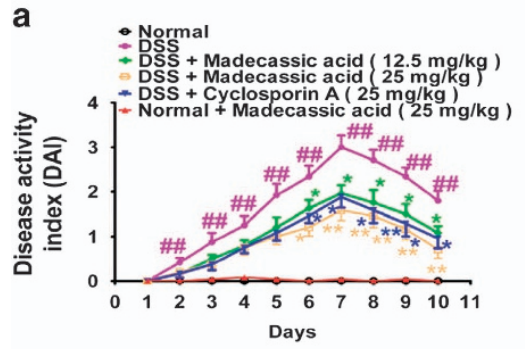

c

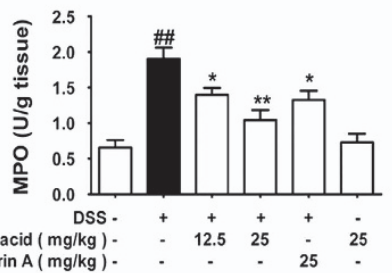

b

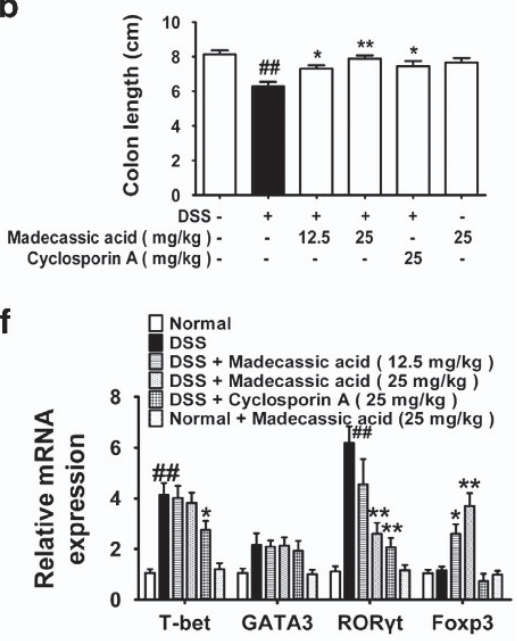

d
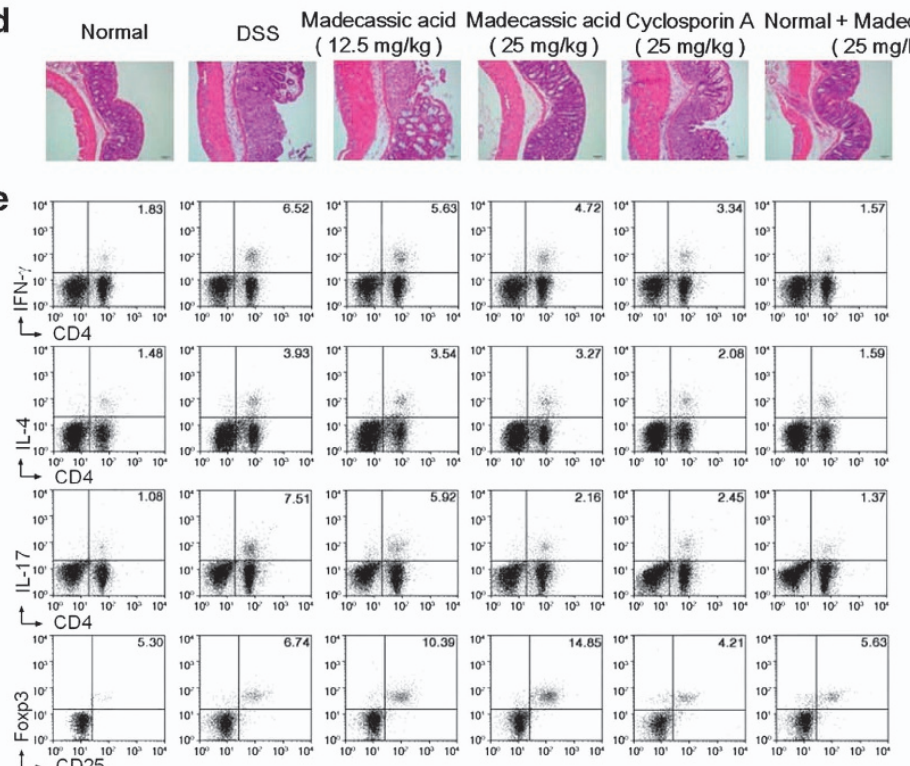

g DSS

\begin{tabular}{|c|c|c|c|c|c|c|c|}
\hline DSS & - & + & + & + & + & - & \\
\hline Madecassic acid $(\mathrm{mg} / \mathrm{kg})$ & - & - & 12.5 & 25 & - & 25 & \\
\hline Cyclosporin A ( $\mathrm{mg} / \mathrm{kg}$ ) & - & - & - & - & 25 & - & \\
\hline RORyt & - & - & - & - & - & - & $58 \mathrm{kDa}$ \\
\hline Foxp3 & & & - & - & - & - & $47 \mathrm{kDa}$ \\
\hline GAPDH & - & - & - & - & - & - & $37 \mathrm{kDa}$ \\
\hline
\end{tabular}

h

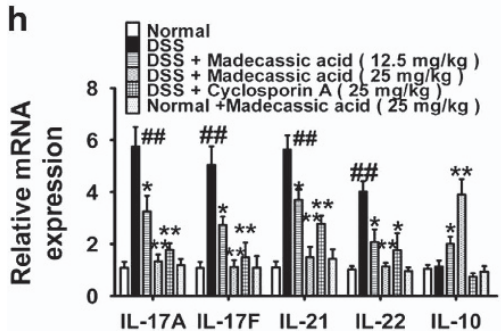

i

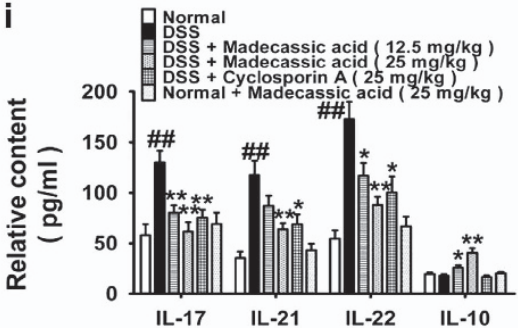

Figure 2 Madecassic acid restored the T helper type 17/regulatory T cell balance in mice with DSS-induced colitis. Mice were fed with $2.5 \%$ DSS for 7 days and then given normal water for 3 days. Madecassic acid $(12.5,25 \mathrm{mg} / \mathrm{kg}$ ) and cyclosporin A (25 mg/kg) were orally administered for 10 days. (a) DAl. (b) Colon length. (c) MPO activity. (d) H\&E staining. (e) The proportions of $\mathrm{CD} 4^{+} \mathrm{IFN}-\gamma^{+} \mathrm{T}$ cells, CD4 $4^{+} \mathrm{IL}-4^{+} \mathrm{T}$ cells, $\mathrm{CD} 4^{+} \mathrm{IL}-17^{+} \mathrm{T}$ cells and $\mathrm{CD} 4^{+} \mathrm{CD} 25^{+} \mathrm{Foxp} 3^{+} \mathrm{T}$ cells were detected by flow cytometry. (f and $\mathrm{g}$ ) The mRNA and protein levels of transcription factors T-bet, GATA-3, ROR $\gamma$ t and Foxp3 in the colons were evaluated by real-time PCR and western blot. (h and i) The levels of IL-17A, IL-17F, IL-21, IL-22 and IL-10 were assessed by enzyme-linked immunosorbent assay and real-time PCR. GAPDH was used as a cytoplasm marker. The data were expressed as means \pm S.E.M., $n=5-8 .{ }^{\# \#} P<0.01$ versus normal group; ${ }^{*} P<0.05,{ }^{* *} P<0.01$ versus DSS group 
malonyl-CoA, enhances and inhibits the polarization of Th17 and Treg cells, respectively. ${ }^{27,28}$ To identify whether the madecassic acid-induced shift of Th17 toward Treg cells was related to $\mathrm{ACC} 1$ inhibition, we evaluated its effect on ACC1 expression. Madecassic acid $(3,10 \mu \mathrm{M})$ downregulated ACC1 expression under Th17-polarizing conditions
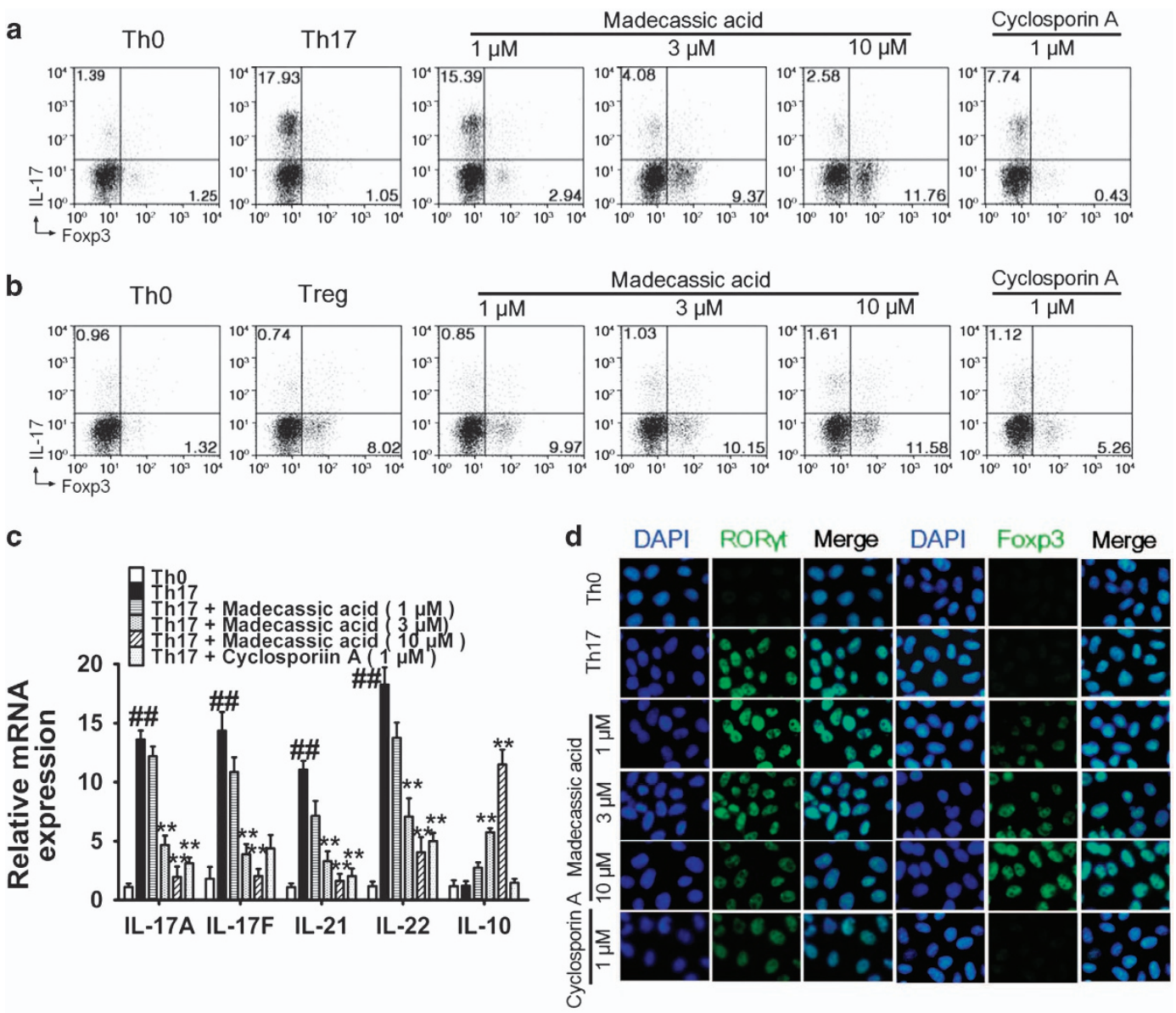

e

Th17 condition
Madecassic acid
Cyclosporin A (
RORYt
Foxp3
p-AKT
AKT
p-JAK2
JAK2
p-STAT3
STAT3
STAT3 (nuclear)
Lamin B1
STAT3 (cytosol)
GAPDH

f

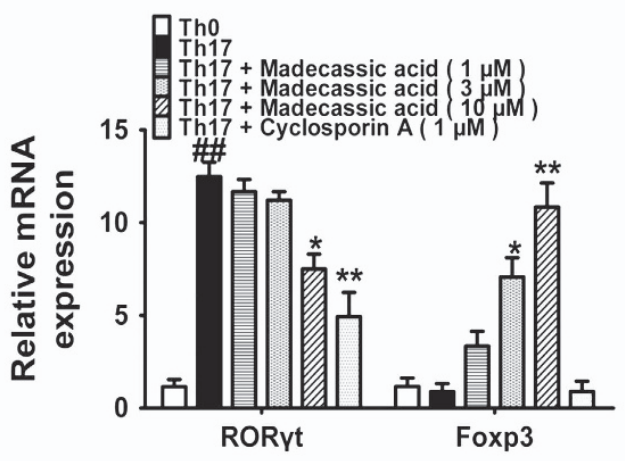

Figure 3 Madecassic acid restored the T helper type 17/regulatory T cell (Th17/Treg) balance by enhancing the shift of of Th17 toward Treg cells. (a) Naive T cells were differentiated under Th17-inducing conditions for 4 days in the presence of madecassic acid $(1,3,10 \mu \mathrm{M})$ or a control. The proportions of CD $4^{+} \mathrm{IL}-17^{+} \mathrm{T}$ cells and CD4 ${ }^{+}$Foxp $3^{+}$ T cells were gated by flow cytometry. (b) Naive T cells were differentiated under Treg-inducing conditions for 4 days in the presence or absence of madecassic acid $(1,3,10 \mu \mathrm{M})$. The proportions of $\mathrm{CD} 4^{+} \mathrm{IL}-17^{+} \mathrm{T}$ cells and $\mathrm{CD} 4^{+} \mathrm{Foxp} 3^{+} \mathrm{T}$ cells were gated by flow cytometry. (c) Naive T cells were cultured with madecassic acid (1,3, 10 $\left.\mu \mathrm{M}\right)$ under Th17inducing conditions for 4 days. The mRNA levels of IL-17A, IL-17F, IL-21, IL-22 and IL-10 were measured by real-time PCR. (d) Naive T cells were cultured with madecassic acid $(1,3,10 \mu \mathrm{M})$ under Th17-inducing conditions for 4 days. The expression of ROR $\gamma$ t and Foxp3 under Th17-polarizing conditions was measured by immunofluorescence. (e) Naive T cells were cultured with madecassic acid $(1,3,10 \mu \mathrm{M})$ under Th17-inducing conditions for 4 days. The protein expression of RORyt and Foxp3, as well as Th17-associated transducers p-AKT, AKT, p-STAT3, STAT3, p-JAK2 and JAK was examined by western blot. (f) Naive T cells were cultured with madecassic acid (1,3, $10 \mu \mathrm{M})$ under Th17inducing conditions for 4 days. The mRNA expression of RORyt and Foxp3 was analyzed by real-time PCR. GAPDH was used as a cytoplasm marker; Lamin B1 was used as a nuclear marker. The data were expressed as means \pm S.E.M., $n=3 .{ }^{\sharp} P<0.05,{ }^{\# \#} P<0.01$ versus Th0 group; ${ }^{*} P<0.05,{ }^{* *} P<0.01$ versus Th17 group 
a

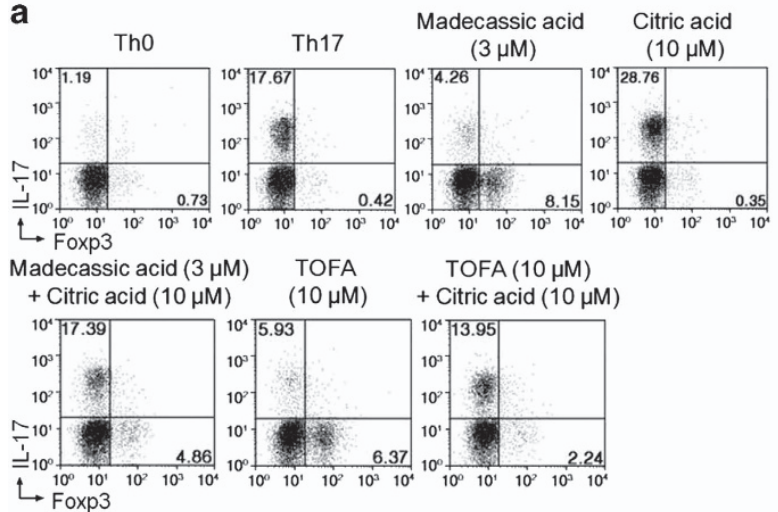

C

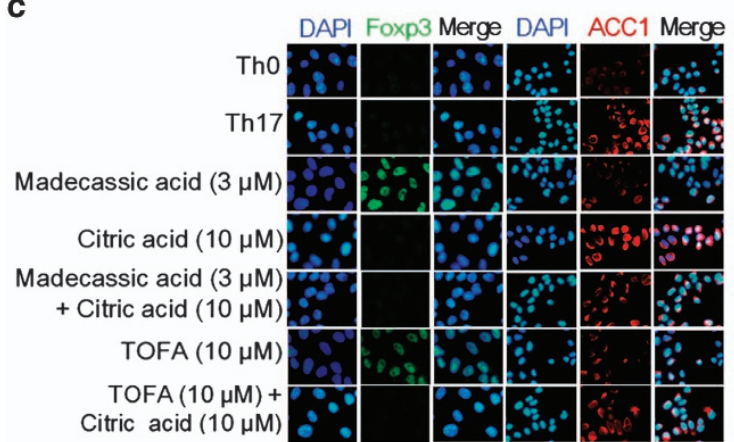

e

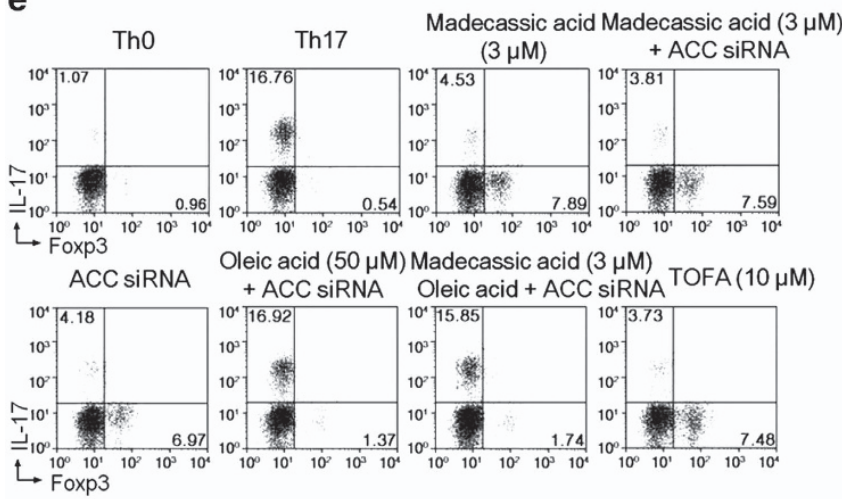

f

Th17 condition
ACC siRNA
Madecassic acid $(\mu \mathrm{M})$
Oleic acid $(\mu \mathrm{M})$
TOFA $(\mu \mathrm{M})$
Foxp3

GAPDH

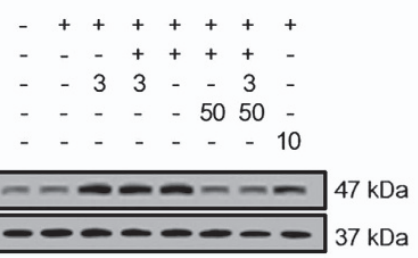

b

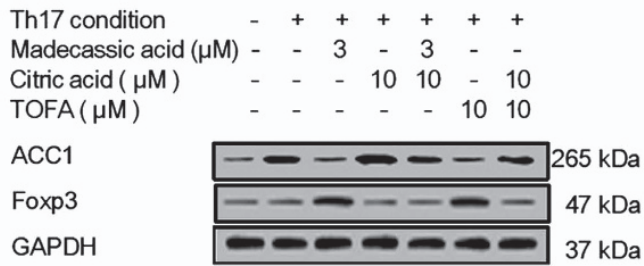

d

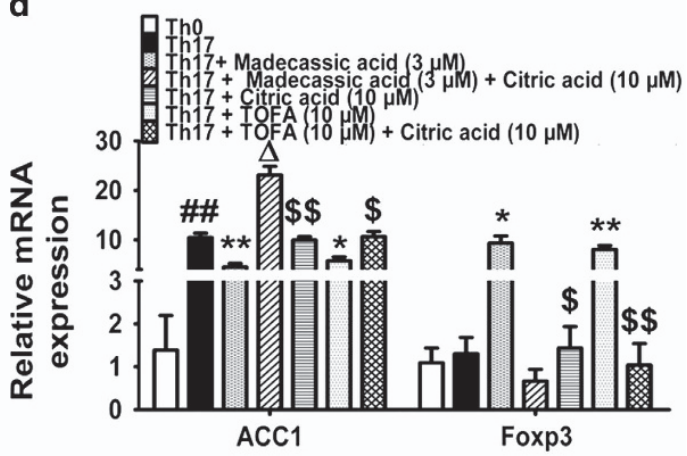

g

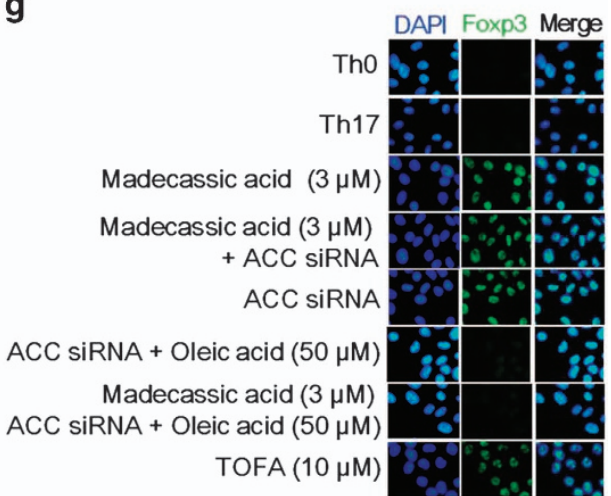

h

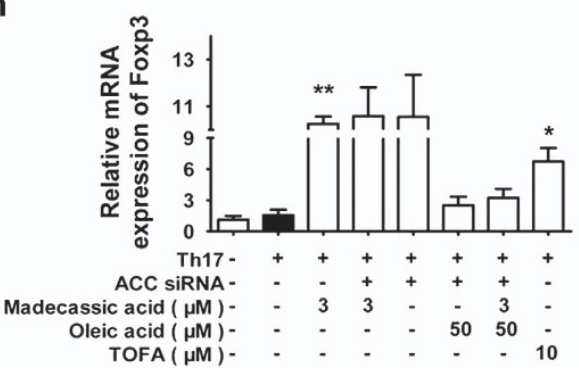

Figure 4 Madecassic acid facilitated the shift of T helper type 17 (Th17) toward regulatory T cells by inhibiting ACC1 expression. (a) Naive T cells were cultured with madecassic acid $(3 \mu \mathrm{M})$ and ACC1 activator citric acid $(10 \mu \mathrm{M})$ under Th17-inducing conditions for 4 days. The proportions of CD4 ${ }^{+} \mathrm{IL}-17^{+} \mathrm{T}$ cells and CD4 $4^{+} \mathrm{Foxp} 3^{+} \mathrm{T}$ cells were detected by flow cytometry. (b-d) Naive T cells were cultured with madecassic acid $(3 \mu \mathrm{M})$ and ACC1 activator citric acid (10 $\mu \mathrm{M})$ under Th17-inducing conditions for 4 days. The mRNA and protein expression of Foxp3 and ACC1 was analyzed by western blot, immunofluorescence and real-time PCR. (e) Naive T cells were cultured with madecassic acid $(3 \mu \mathrm{M})$, oleic acid $(50 \mu \mathrm{M})$ and ACC1 siRNA under Th17-polarizing conditions for 4 days. The proportions of CD4 $4^{+} \mathrm{IL}-17^{+} \mathrm{T}$ cells and CD4 ${ }^{+}$Foxp3 ${ }^{+} \mathrm{T}$ cells were detected by flow cytometry. (f-h) Naive T cells were cultured with madecassic acid $(3 \mu \mathrm{M})$, oleic acid $(50 \mu \mathrm{M})$ and ACC1 siRNA under Th17-polarizing conditions for 4 days. The relative expression of Foxp3 was analyzed by western blot, immunofluorescence and real-time PCR. GAPDH was used as a cytoplasm marker. The data were expressed as means \pm S.E.M., $n=3$. ${ }^{\#} P<0.05,{ }^{\# \#} P<0.01$ versus Th0 group; ${ }^{\star} P<0.05$, ${ }^{\star \star} P<0.01$ versus Th17 group; $\Delta P<0.05$ versus Th17 group; ${ }^{\$} P<0.05$, ${ }^{\$ \$} P<0.01$ versus citric acid group 
(Supplementary Figure S3a), and oral madecassic acid $(12.5,25 \mathrm{mg} / \mathrm{kg}$ ) suppressed the ACC1 expression in the colons of DSS-treated mice (Supplementary Figure S3b). In addition, the shift of Th17 toward Treg cells induced by madecassic acid was diminished by ACC1 activator citric acid under Th17-polarizing conditions (Figure $4 a$ and Supplementary Figure S1). Consistently, citric acid reversed the madecassic acid-induced downregulation of Th17 cellspecific cytokines expression, the upregulation of IL-10 expression (Supplementary Figure S3c) and the enhancement of Foxp3 expression under Th17-polarizing conditions (Figures $4 b-d$ and Supplementary Figure S1). The suppression of ACC1 expression by madecassic acid was also diminished by citric acid (Figures 4b-d and Supplementary Figure S1). These findings suggested that madecassic acid enhanced the shift of Th17 toward Treg cells by suppressing the ACC1 expression.

To identify whether the shift of Th17 toward Treg cells induced by madecassic acid take place through the downregulation of $A C C 1$ catalytic products, naive $C D 4^{+} T$ cells were subjected to ACC1 depletion or treated with oleic acid (an ACC1 catalytic product) under Th17-polarizing conditions. Interestingly, the shift of Th17 toward Treg cells by madecassic acid was not affected by ACC1 depletion but inhibited by oleic acid (Figure 4e and Supplementary Figure S1). In addition, madecassic acid-induced decrease in Th17 cell-specific cytokines expression and increase in IL-10 expression were inhibited by oleic acid under ACC1 depletion (Supplementary Figure S3d). The high level of Foxp3 expression induced by madecassic acid was also blunted by oleic acid (Figures $4 \mathrm{f}-\mathrm{h}$ and Supplementary Figure S1). These findings suggested that madecassic acid shifted Th17 toward Treg cells through downregulation of ACC1 expression.

\footnotetext{
Madecassic acid inhibited ACC1 expression and shifted Th17 toward Treg cells by activating adenosine monophosphate-activated protein kinase (AMPK). ACC1-mediated fatty acid synthesis can be inhibited by the activation of AMPK, a cellular energy sensor. ${ }^{29,30}$ Madecassic acid $(3,10 \mu \mathrm{M})$ enhanced the phosphorylation of AMPK under Th17-polarizing conditions (Figure 5a and Supplementary Figure S1) and in the colons of DSS-induced mice (Supplementary Figure S3b). The inhibitory effect of madecassic acid on ACC1 expression was weakened by AMPK antagonist compound $\mathrm{C}$ and siRNA under Th17-polarizing conditions (Figures $5 e$ and f). In contrast, madecassic acidinduced activation of AMPK was not affected by ACC1 siRNA or oleic acid (Figure $5 b$ and Supplementary Figure S1), suggesting that madecassic acid suppressed the ACC1 expression through the activation of AMPK. Furthermore, both compound $\mathrm{C}$ and AMPK siRNA inhibited madecassic acid-induced shift of Th17 toward Treg cells (Figures 5c, d and Supplementary Figure S1), regulation of Th17 cellspecific cytokines and IL-10 expression (Supplementary Figures $\mathrm{S} 3 \mathrm{e}$ and $\mathrm{f}$ ), the increase in Foxp3 expression and the decrease in ACC1 expression (Figures $5 \mathrm{e}-\mathrm{h}$ and Supplementary Figure S1). These findings suggested that madecassic acid enhanced the shift of Th17 toward Treg cells by downregulating the ACC1 expression via activation of AMPK.
}

Madecassic acid promoted AMPK activation in the shift of Th17 toward Treg cells by activating PPARy. Madecassic acid $(3,10 \mu \mathrm{M})$ promoted the translocation of PPARy from the cytoplasm to the nucleus under Th17-polarizing conditions (Supplementary Figure S4a). It also promoted PPARy nuclear translocation in the colons of DSS-induced mice (Supplementary Figure S4b). To verify the key role that PPARy has in the madecassic acid-induced shift of Th17 toward Treg cells under Th17-polarizing conditions, PPARy antagonists GW9662 and T0070907 as well as PPARy siRNA were used. The results showed that either pharmacological antagonism or knockdown of PPARy inhibited madecassic acid-induced shift of Th17 toward Treg cells (Figures 6a and b) and regulation of Th17 cell-specific cytokines and IL-10 expression (Supplementary Figures S4C and d) and Foxp3 expression (Figures $6 c-f$ and Supplementary Figure S1). These data revealed that madecassic acid promoted the shift of Th17 toward Treg cells by activating PPARy.

Further studies showed that the madecassic acid-induced AMPK activation and ACC1 inhibition were inhibited by GW9662, T0070907 and PPARy siRNA (Figures 6c-f and Supplementary Figure S1) Conversely, neither pharmacological antagonism nor knockdown of AMPK or ACC1 affected the translocation of PPARy induced by madecassic acid (Figure $6 \mathrm{~g}$ and Supplementary Figures S1 and S4e-g). These findings revealed that PPAR $y$ mediated the activation of AMPK and subsequent inhibition of ACC1 expression induced by madecassic acid in the shift of Th17 toward Treg cells.

Madecassic acid acted as a PPARy agonist. Madecassic acid $(3,10 \mu \mathrm{M})$ elevated the expression of PPAR $y$-responsive genes fatty acid translocase (CD36) and lipoprotein lipase (LPL) in T lymphocytes (EL-4 cells) (Figures $7 \mathrm{a}, \mathrm{b}$ and Supplementary Figure S1) without marked effect on PPARaand PPAR $\beta$-responsive genes (Supplementary Figure S5a). Madecassic acid $(12.5,25 \mathrm{mg} / \mathrm{kg})$ also induced the expression of CD36 and LPL in the colons of DSS-induced mice (Supplementary Figures S5b and c). These data implied that madecassic acid selectively promoted the expression of PPARy-responsive genes. GW9662, T0070907 and PPARy siRNA inhibited the effect of madecassic acid on CD36 and LPL expression (Figures 7c-f and Supplementary Figure S1), suggesting that madecassic acid enhanced CD36 and LPL expression in a PPAR $y$-dependent manner. To determine whether the increased CD36 and LPL expression induced by madecassic acid was a result of de novo RNA synthesis or protein synthesis, EL-4 cells were pretreated with RNA polymerase inhibitor actinomycin $D$ (Act-D) or protein synthesis inhibitor cycloheximide (CHX). Both Act-D and $\mathrm{CHX}$ almost completely abolished the madecassic acidinduced increase in the CD36 and LPL expression (Supplementary Figures S5d-f), indicating that the effect of madecassic acid on PPARy-responsive genes depended on both RNA and protein synthesis. An in-depth analysis of molecular operating environment (MOE) docking module showed that madecassic acid extended deep into the PPAR $y$-ligand binding domain (LBD) and interacted with hydrophobic residues at Gly 284, where the $\mathrm{H}$-bonds improved the binding affinity of madecassic acid to PPARy 
(Figure 7g). A time-resolved fluorescence resonance energy transfer (TR-FRET) assay further demonstrated that madecassic acid bounded to PPARy with a kinetic inhibition constant $\left(K_{\mathrm{i}}\right)$ of $1.61 \mu \mathrm{M}$ (Figure $7 \mathrm{~h}$ ). An electrophoretic mobility shift assay (EMSA) was performed to verify that madecassic acid indeed induced the binding of PPARy to the PPAR response element (PPRE) sequence, which could be diminished by GW9662 and T0070907 (Figure 7i). A luciferase reporter assay showed that madecassic acid markedly enhanced PPRE reporter activity in EL-4 cells, which was reduced by GW9662 and T0070907 (Figure 7j). These data suggested that madecassic acid was a potential agonist of PPAR $y$. a

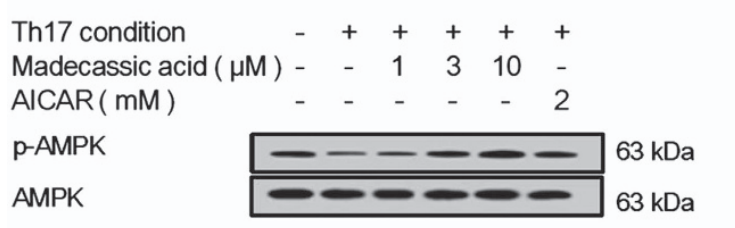

b

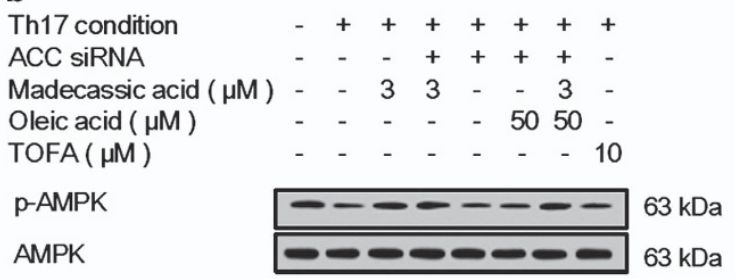

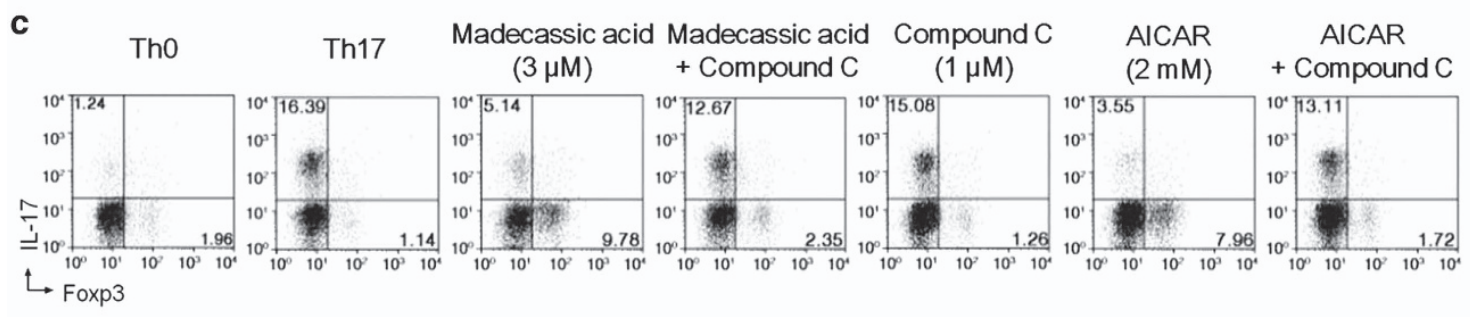

d
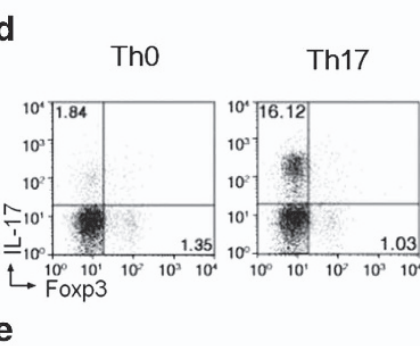

e

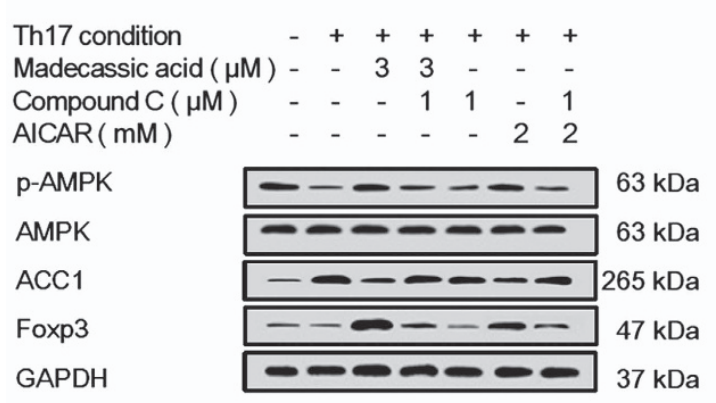

f

Th17 condition $\quad-++++++$

Madecassic acid $(\mu \mathrm{M})$ - 33 - 3 -

AMPK SiRNA $\quad-\quad-\quad+\quad+\quad-+$

$\operatorname{AICAR}(\mathrm{mM}) \quad-\quad-\quad-22$

ACC1

Foxp3

GAPDH

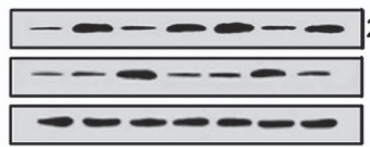

$265 \mathrm{kDa}$

$47 \mathrm{kDa}$

$37 \mathrm{kDa}$
Madecassic acid
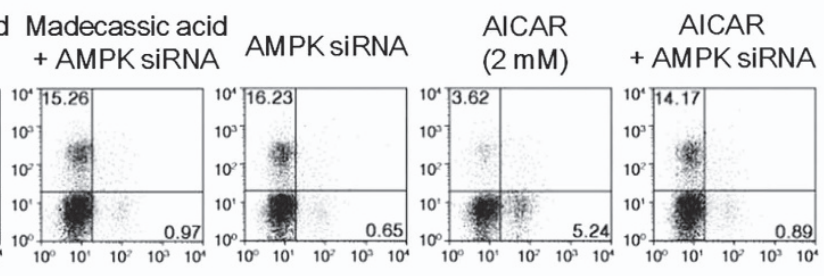

g

Madecassic acid $(3 \mu \mathrm{M})$

Madecassic acid ( $3 \mu \mathrm{M})$

+ Compound C $(1 \mu \mathrm{M})$

Compound C $(1 \mu \mathrm{M})$

$\operatorname{AICAR}(2 \mathrm{mM})$

$\operatorname{AICAR}(2 \mathrm{mM})$

+ Compound C $(1 \mu \mathrm{M})$

DAPI Foxp3 Merge DAPI ACC1 Merge

h

Madecassic acid $(3 \mu \mathrm{M})$

Madecassic acid $(3 \mu \mathrm{M})$ + AMPK siRNA

AMPK SiRNA

$\operatorname{AICAR}(2 \mathrm{mM})$

$\operatorname{AICAR}(2 \mathrm{mM})$

+ AMPK siRNA

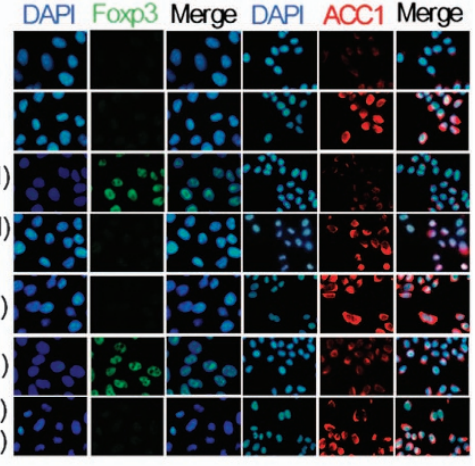

DAPI Foxp3 Merge DAPI ACC1 Merge

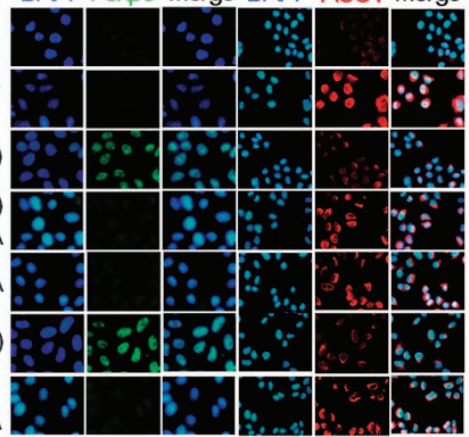


Madecassic acid recovered Th17/Treg balance in mice with DSS-induced colitis through PPARy/AMPK/ACC1 pathway. To further ascertain the causal link between PPARy activation, AMPK/ACC1 signaling regulation, Th17/ Treg balance restoration and colitis amelioration by madecassic acid, madecassic acid was orally administered in combination with GW9662 in mice fed with DSS. GW9662 counteracted the madecassic acid-induced enhancement of CD36 and LPL expression in the colons (Figure 8a and Supplementary Figure S1). As expected, madecassic acid promoted the activation of AMPK and inhibited the expression of ACC1 in the colons, and these effect were markedly weakened by GW9662 (Figure 8b and Supplementary Figure S1). The decreased percentage of Th17 cells and the increased percentage of Treg cells induced by madecassic acid in the colons were reversed by GW9662 (Figure 8c and Supplementary Figure S1). Moreover, GW9662 weakened the downregulation of Th17 cell-specific cytokine expression and the upregulation of IL-10 expression by madecassic acid in the colons (Figure 8d) and diminished madecassic acid-induced upregulation of Foxp3 expression (Figure $8 \mathrm{~b}$ and Supplementary Figure $\mathrm{S} 1$ ) and protection against colitis (Figures $8 \mathrm{e}-\mathrm{h}$ ). These results revealed that madecassic acid exerted anti-colitis effect by restoring the Th17/Treg balance by shifting Th17 toward Treg cells via the PPAR $\gamma / A M P K / A C C 1$ pathway.

\section{Discussion}

Four main pentacyclic triterpenes in $C$. asiatica could alleviate DSS-induced colitis in mice by oral administration. Among them, madecassoside conferred superior protection against the pathological lesions in the colon. When rectally administered, madecassoside but not madecassic acid was unable to protect against colitis. As madecassoside was the most abundant triterpene in $C$. asiatica and it would rapidly dehydrate into madecassic acid in the intestines, we postulated that madecassoside was the primary active ingredient of C. asiatica for ameliorating colitis, and it functioned through madecassic acid.

DSS application results in mucosal barrier damage with associated microbial translocation, inflammation and immune activation. ${ }^{31}$ The excessive activation of the immune system can be primarily attributed to Th17/Treg imbalance, resulting from the increase of functionally active Th17 cells and a lack of immune-suppressive Treg cells. ${ }^{32-34}$ Thus the restoration of
Th17/Treg balance is conducive to the immune homeostasis and may be an effective therapeutic strategy for UC. In this study, madecassic acid recovered the Th17/Treg balance by decreasing the percentage of Th17 cells and promoting the expansion of Treg cells without affecting the percentages of Th1 and Th2 cells in mice fed with DSS.

Restoring the Th17/Treg balance could be achieved by inhibiting Th17 differentiation, promoting Treg differentiation and shifting between Th17 and Treg cells. ${ }^{35-38}$ In vitro, madecassic acid decreased the proportion of $\mathrm{CD} 4^{+} \mathrm{IL}-17^{+}$ $\mathrm{T}$ cells and markedly increased the proportion of $\mathrm{CD} 4^{+} \mathrm{Foxp} 3^{+}$ T cells and the expression of Foxp3 and IL-10 under Th17polarizing conditions. However, it did not affect the differentiation of Treg cells under Treg-polarizing conditions. It was suggested that madecassic acid might restore Th17/Treg balance by enhancing the shift of Th17 toward Treg cells.

The shift of Th17 toward Treg cells can be induced by energy metabolism regulators. ${ }^{39-41}$ Activated T cells experience an enormous metabolic switch to meet the demands of cell polarization and implementation of their function. ${ }^{42,43}$ Downregulation or deletion of ACC1 inhibits the plasticity of human and mouse Th17 cells, characterized by promoting the shift of Th17 toward Treg cells. ${ }^{27}$ Madecassic acid was shown to enhance the shift of Th17 toward Treg cells by downregulating the expression of $A C C 1$, and it would lose the ability in the presence of oleic acid, suggesting that ACC1-mediated de novo fatty acid synthesis was critical for madecassic acidmediated Th17/Treg rebalance.

The activation of AMPK, an upstream regulator of ACC1, will reduce Th17 cell differentiation and favor Treg cell development. ${ }^{44-46}$ In this study, madecassic acid was shown to accelerate AMPK activation, and compound $C$ abolished madecassic acid-induced activation of AMPK and downregulation of ACC1 expression under Th17-polarizing conditions, indicating that madecassic acid downregulated the ACC1 expression by activating AMPK in Th17 cells. Moreover, both compound $C$ and AMPK siRNA reversed the shift induced by madecassic acid. These findings revealed that madecassic acid promoted an ACC1-mediated shift of Th17 toward Treg cells via activating AMPK under Th17-polarizing conditions.

PPARy, a ligand-dependent nuclear receptor that has vital roles in adipogenesis, glucose metabolism and immune modulation, is highly expressed in the colon. ${ }^{47-49}$ Intensive preclinical studies have highlighted that the activation of PPARy conferred protection against colitis in mice. ${ }^{50-52}$ Madecassic acid was shown to activate AMPK in a

\footnotetext{
Figure 5 Madecassic acid regulated ACC1-mediated shift of T helper type 17 (Th17) toward regulatory T cells via the activation of AMPK. Naive T cells were cultured with or without compound $\mathrm{C}$ and AMPK siRNA under Th17-inducing conditions in the presence of madecassic acid for 4 days. (a) Naive T cells were treated with madecassic acid (1, 3 , $10 \mu \mathrm{M}$ ) under Th17-inducing conditions for 4 days. The protein expression of p-AMPK was analyzed by western blot. (b) Naive T cells were cultured with madecassic acid ( $3 \mu \mathrm{M}$ ), oleic acid $(50 \mu \mathrm{M})$ and ACC1 siRNA under Th17-polarizing conditions for 4 days. The protein expression of p-AMPK was measured by western blot. (c and d) Naive T cells were cultured with madecassic acid $(3 \mu \mathrm{M})$, compound $\mathrm{C}(1 \mu \mathrm{M})$ and AMPK siRNA under Th17-polarizing conditions for 4 days. The proportions of CD4 ${ }^{+} \mathrm{IL}-17^{+} \mathrm{T}$ cells and $\mathrm{CD} 4^{+}$ Foxp $3^{+} \mathrm{T}$ cells were evaluated by flow cytometry. (e) Naive T cells were cultured with madecassic acid $(3 \mu \mathrm{M})$ and compound $\mathrm{C}(1 \mu \mathrm{M})$ under Th17-polarizing conditions for 4 days. The relative expression of p-AMPK, AMPK, ACC1 and Foxp3 was analyzed by western blot. (f) Naive T cells were cultured with madecassic acid ( $3 \mu \mathrm{M})$ and AMPK siRNA under Th17-polarizing conditions for 4 days. The relative expression of ACC1 and Foxp3 was analyzed by western blot. (g) Naive T cells were cultured with madecassic acid $(3 \mu \mathrm{M})$ and compound $\mathrm{C}(1 \mu \mathrm{M})$ under Th17-polarizing conditions for 4 days. The relative expression of ACC1 and Foxp3 was analyzed by immunofluorescence. (h) Naive T cells were cultured with madecassic acid $(3 \mu \mathrm{M})$ and AMPK siRNA under Th17-polarizing conditions for 4 days. The relative expression of ACC1 and Foxp3 was analyzed by immunofluorescence. GAPDH was used as a cytoplasm marker. The data were expressed as the means \pm S.E.M., $n=3 .{ }^{\#} P<0.05$, ${ }^{\# \#} P<0.01$ versus Th0 group; ${ }^{*} P<0.05$, ${ }^{* *} P<0.01$ versus Th17 group; ${ }^{\$} P<0.05,{ }^{\$ \$} P<0.01$ versus madecassic acid group
} 
PPAR $y$-dependent manner and induce the AMPK/ACC1mediated shift of Th17 toward Treg cells by activating PPAR $y$. In contrast, inhibition or depletion of AMPK did not inhibit madecassic acid-induced translocation of PPARy into the nucleus. The findings suggested that AMPK might be a downstream effector of PPAR $y$.
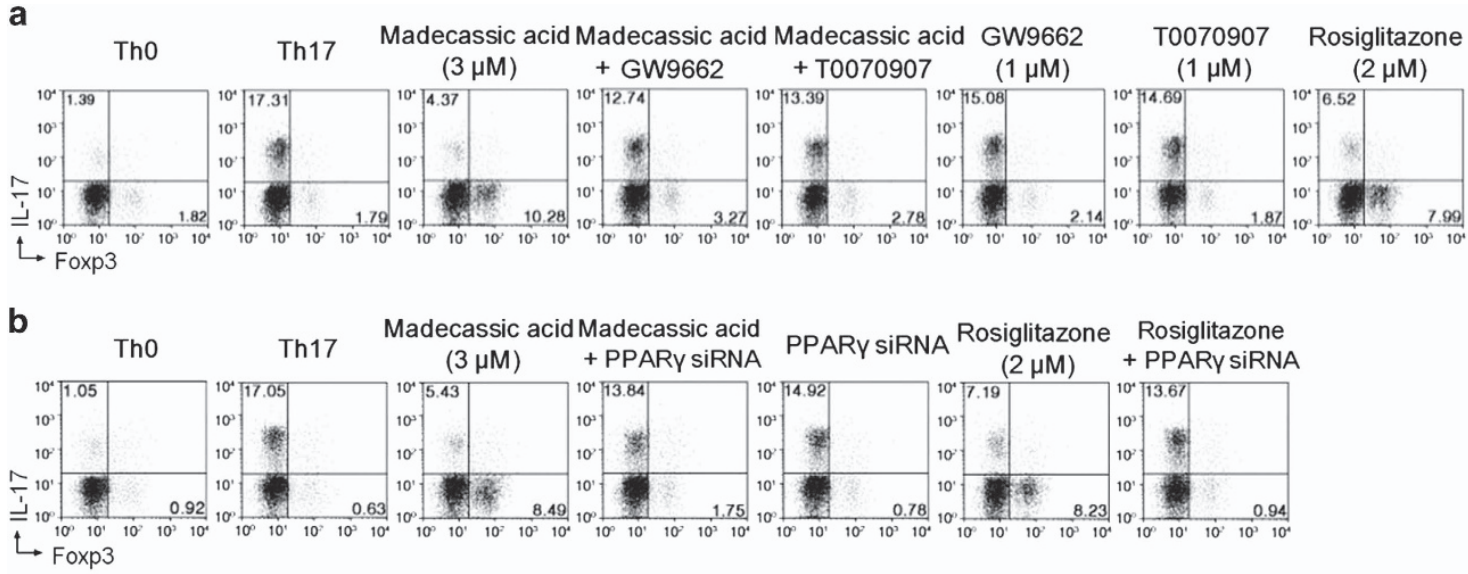

c

\section{DAPI Foxp3 Merge DAPI ACC1 Merge DAPI PPARy Merge}

$$
\begin{array}{r}
\text { Madecassic acid }(3 \mu \mathrm{M}) \\
\text { Madecassic acid }(3 \mu \mathrm{M}) \\
+ \text { GW9662 }(1 \mu \mathrm{M}) \\
\text { Madecassic acid }(3 \mu \mathrm{M}) \\
+ \text { T0070907 }(1 \mu \mathrm{M}) \\
\text { GW9662 }(1 \mu \mathrm{M}) \\
\text { T0070907 }(1 \mu \mathrm{M})
\end{array}
$$

Rosiglitazone $(2 \mu \mathrm{M})$

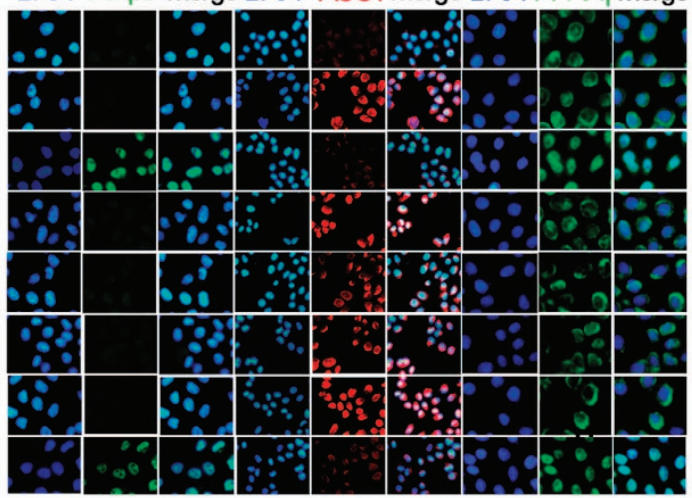

d

Madecassic acid $(3 \mu \mathrm{M})$

Madecassic acid $(3 \mu \mathrm{M})$ + PPARY siRNA

\begin{tabular}{|c|c|}
\hline p-AMPK & $-\cdots-\cdots$ \\
\hline AMPK & $-\infty-\cdots-\cdots$ \\
\hline ACC1 & $-\cdots-\cdots$ \\
\hline Foxp3 & $-\cdots-\cdots$ \\
\hline GAPDH & $-\infty-\infty-\infty$ \\
\hline
\end{tabular}

PPARY SIRNA

Rosiglitazone $(2 \mu \mathrm{M})$

Rosiglitazone $(2 \mu \mathrm{M})$ + PPARY siRNA

f

$\begin{array}{llllllll}\text { Th17 condition } & - & + & + & + & + & + & + \\ \text { Madecassic acid }(\mu \mathrm{M}) & - & - & 3 & 3 & - & - & - \\ \text { PPAR y siRNA } & - & - & - & + & + & - & + \\ \text { Rosiglitazone }(\mu \mathrm{M}) & - & - & - & - & - & 2 & 2\end{array}$ e

Th17 condition -+++++++ Madecassic acid $(\mu \mathrm{M})$ - $\quad-\quad \begin{array}{lllll}3 & 3 & 3 & - & -\end{array}$

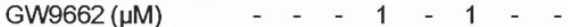
T0070907 $(\mu \mathrm{M}) \quad-\quad-\quad-\quad-1-1$ -

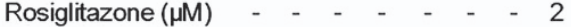
p-AMPK AMPK ACC1 Foxp3 GAPDH

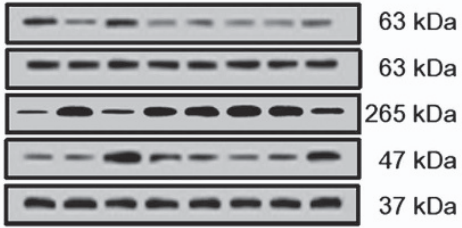
g

Th17 condition $\quad-++++++$ Madecassic $\operatorname{acid}(\mu \mathrm{M})$ - $\quad-33-\quad-$ AMPK SIRNA $\quad-\quad-\quad+\quad+\quad+$ $\operatorname{AICAR}(\mathrm{mM}) \quad-\quad-\quad-22$ PPARY (cytosol) GAPDH PPARY (nuclear) Lamin B1

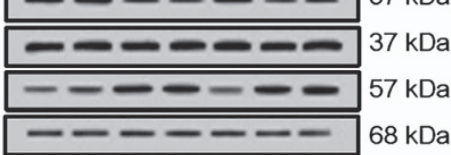


There are many reports which indicate that high-affinity synthetic ligands of PPAR $y$, such as thiazolidinediones, have immunoprotective roles in experimental colitis. ${ }^{53-55}$ Similar to rosiglitazone, madecassic acid promoted the expression of PPARy-responsive genes CD36 and LPL, induced PPARy translocation from cytoplasm to nucleus and the binding of PPARy to a reporter gene, which could be diminished by PPARy antagonists or PPAR $y$ siRNA. These results indicated that madecassic acid acted as a PPARy agonist in regulating the AMPK/ACC1-mediated Th17/Treg balance.

In conclusion, madecassoside was the major active ingredient of $C$. asiatica in alleviating DSS-induced colitis, and it acted through the intestinal metabolite madecassic acid. Madecassic acid exerted anti-colitis effect by restoring Th17/Treg balance via the PPARy/AMPK/ACC1 pathway.

\section{Materials and Methods}

Reagents. Madecassoside (C48H78020, molecular weight (MW): 975.12, purity $\geq 98 \%$ ), asiaticoside (C48H78019, MW: 959.12, purity $\geq 98 \%$ ), madecassic acid (C30H48O6, MW: 504.70 , purity $\geq 98 \%)$ and asiatic acid (C3OH48O5, MW: 488.70, purity $\geq 98 \%$ ) purchased from Zelang Pharmaceutical Technology Co., Ltd (Zelang, Nanjing, China) were suspended in $0.5 \%$ sodium carboxy methyl cellulose (CMC-Na) for oral or rectal administration. Madecassic acid was dissolved in dimethyl sulfoxide (DMSO) and diluted to $10 \mathrm{mM}$ stock solution in phosphatebuffered saline for in vitro studies. Cyclosporin A was received from Ipsen Pharma (Ethypharm, Houdan, France). DSS (MW: $36-50 \mathrm{kDa}$ ) was obtained from MP Biomedicals Inc. (Irvine, CA, USA). Mouse IL-17, IL-21, IL-22 and IL-10 ELISA kits were purchased from Dakewe Biotech Co., Ltd (Shenzhen, China). MPO activity assay kit was obtained from Nanjing Jiancheng Bioengineering Institute (Jiancheng, Nanjing, China). Murine IL-6, IL-23, IL-1 $\beta$ and human IL-2, TGF- $\beta 1$ were provided by R\&D Systems (Minneapolis, CA, USA). Mouse $\mathrm{CD}^{+} \mathrm{CD}^{+} \mathrm{L}^{+} \mathrm{T}$ Cell Isolation Kit was purchased from Miltenyi Biotec Inc. (Santa Barbara, CA, USA). EL-4 mouse lymphoma cell line was provided by American Type Culture Collection (ATCC, Manassas, VA, USA). FITC-anti-CD4, PE-anti-IL-17A, PE-anti-CD25, APC-anti-IL-4, PE-anti-IFN- $\gamma$ and APC-anti-Foxp3 were purchased from eBioscience, Inc. (Affymetrix, San Diego, CA, USA). Purified anti-mouse $\mathrm{CD} 3 \varepsilon$ and anti-mouse CD28 antibodies were provide by BD Biosciences, Inc. (San Jose, CA, USA). GW9662 and T0070907 (PPAR $\gamma$ antagonists), 5-aminoimidazole-4-carboxamide1$\beta$-D-ribofuranoside (AICAR, AMPK activator), 5-(tetradecyloxy)-2-furoic acid (TOFA, ACC1 inhibitor), citric acid (ACC1 activator), oleic acid (ACC1 catalytic product), compound C (AMPK inhibitor), rosiglitazone (PPAR $\gamma$ agonist) and cycloheximide (CHX, protein synthesis inhibitor) were purchased from Sigma Aldrich (St. Louis, MO, USA). Act-D (RNA polymerase inhibitor) was purchased from Zhejiang Haizheng Pharmaceutical Co. Ltd (Haizheng, Hangzhou, China). TRIzol, Dye DAPI and Lipofectamine 2000 reagents were purchased from Invitrogen (Carlsbad, CA, USA). ACC1 siRNA, AMPK siRNA and PPAR $\gamma$ siRNA (m) were supplied by Santa Cruz Biotechnology (Santa Cruz, CA, USA). Luciferase Reporter Gene Assay Kit was from Promega Biotech Co., Ltd. (Madison, WI, USA). LanthaScreen TR-FRET PPAR $\gamma$ Competitive Binding Assay was purchased from Thermo Fisher Co., Ltd (Thermo Fisher, Waltham, MA, USA). Primary antibodies against the following targets: ACC1, p-AMPK, AMPK, PPAR $\gamma$, p-STAT3, p-STAT5, STAT3, STAT5, Smad3, p-Smad3, AKT, p-AKT, JAK2, p-JAK2, Foxp3, RORyt, CD36, LPL, Lamin B1 and glycer-aldehyde-3-phosphate dehydrogenase (GAPDH) were purchased from BioWorld (Atlanta, GA, USA). Ace qPCR SYBR Green Master Mix was obtained from Vazyme Co., Ltd (Vazyme, Nanjing, China). Other analytical reagents were obtained from Sino pharm Chemical Reagent Co. Ltd (Nanjing, China).

Animals. Female C57BL/6 mice, 6-8-week-old, were purchased from the Comparative Medicine Center of Yangzhou University (Yangzhou, China). The mice were raised in standard animal cages under specific pathogen-free conditions in the animal facility at the Central Animal Care Services. All animal experiments were performed in accordance with the Guide for the Care and Use of Laboratory Animals.

Induction and assessment of DSS-induced colitis and drug administration. Colitis was induced by an administration of $2.5 \%$ (w/v) DSS that dissolved in sterile distilled water ad libitum for 7 days followed by normal drinking water for 3 days. ${ }^{56}$ Madecassoside $(50 \mathrm{mg} / \mathrm{kg})$, asiaticoside $(50 \mathrm{mg} / \mathrm{kg})$, madecassic acid $(25 \mathrm{mg} / \mathrm{kg})$, asiatic acid $(25 \mathrm{mg} / \mathrm{kg}$ ) and cyclosporin A (25 mg/kg) were orally or rectally administered daily throughout the experiment. GW9662 $(1 \mathrm{mg} / \mathrm{kg}$ ) was administered by an intraperitoneal injection daily for 10 days. Body weight, diarrhea and hematochezia were measured daily after the induction of colitis. The DAI was accounted by the average value of the following three elements: (a) weight loss ( $0=$ none; $1=1-5 \% ; 2=5-10 \% ; 3=10-15 \% ; 4=$ over $15 \%)$; (b) diarrhea scores $(0=$ normal; $2=$ loose stools; $4=$ diarrhea); and (c) blood stool scores $(0=$ normal; $2=$ hemoccult; $4=$ gross bleeding).

After the induction of colitis with DSS, the colon length was measured and then the colon was fixed in 4\% PBS-buffered formaldehyde as a roll and embedded in paraffin. The $5 \mathrm{~mm}$ tissue sections were stained with hematoxylin and eosin. Histological scores were graded as follows: (a) severity of inflammation: $0=$ none; $1=$ slight; $2=$ moderate; $3=$ severe; $($ b) sites of inflammation: $0=$ none; $1=$ mucosa; $2=$ mucosa and submucosa; $3=$ transmural; and (c) lesions of crypt: $0=$ none; $1=$ basal $1 / 3$ damaged; $2=$ basal $2 / 3$ damaged; $3=$ only surface epithelium intact; $4=$ entire crypt and epithelium lost. The histological score was assessed by average of the three evaluations with a maximal score of 10 .

Mouse $\mathrm{T}$ cell cultures. Naive $\mathrm{CD} 4^{+} \mathrm{T}$ cells were isolated ex vivo from the spleens and lymph nodes of mice and purified with magnetic beads $\mathrm{CD}^{+} \mathrm{CD}_{2} \mathrm{~L}^{+} \mathrm{T}$ Cell Isolation Kit. The purity of the isolated cells was $>95 \%$. Naive $\mathrm{CD}^{+} \mathrm{T}$ cells were cultured in RPMI 1640, supplemented with 10\% FCS, $500 \mathrm{U}$ penicillin streptomycin and $50 \mu \mathrm{M} \beta$-mercaptoethanol. For Th17 cell induction,

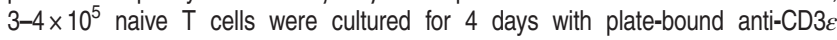
$(10 \mu \mathrm{g} / \mathrm{ml})$, anti-CD28 $(1 \mu \mathrm{g} / \mathrm{ml})$, anti-IFN- $\gamma(5 \mu \mathrm{g} / \mathrm{ml})$, anti-IL-4 $(5 \mu \mathrm{g} / \mathrm{ml})$, rhTGF- $\beta 1$ $(2 \mu \mathrm{g} / \mathrm{ml})$, rmlL-6 $(5 \mu \mathrm{g} / \mathrm{ml})$ and rmlL-1 $\beta(50 \mu \mathrm{g} / \mathrm{ml})$. For Treg cell induction, $2.5 \times 10^{4}$ naive T cells were cultured for 4 days in the presence of plate-bound antiCD3 $\varepsilon(5 \mu \mathrm{g} / \mathrm{ml})$, anti-CD28 $(1 \mu \mathrm{g} / \mathrm{ml})$, rhlL-2 $(200 \mathrm{U} / \mathrm{ml})$ and rhTGF- $\beta 1(1 \mu \mathrm{g} / \mathrm{ml})$. On day 2, rhlL-2 (200 U/ml) was added. ${ }^{28,57}$ Citric acid, TOFA, AICAR, compound C, rosiglitazone, oleic acid, GW9662 and T0070907 were added at the beginning of the cultures. The frequencies of Th17 and Treg cells were detected by flow cytometry.

The EL-4 cells were cultured at $37^{\circ} \mathrm{C}$ under $5 \% \mathrm{CO}_{2}$ atmospheric condition in RPMI 1640 medium with a supplement of $10 \% \mathrm{FBS}, 100 \mathrm{U} / \mathrm{ml}$ streptomycin and $100 \mathrm{U} / \mathrm{ml}$ penicillin. The cells $\left(1 \times 10^{4}\right.$ cells/well) were inoculated into six-well plates

Figure 6 Madecassic acid regulated AMPK/ACC1 via activating PPAR $\gamma$ in the shift of Thelper type 17 (Th17) toward regulatory T cells. Naive T cells were treated with GW9662 $(1 \mu \mathrm{M})$, T0070907 $(1 \mu \mathrm{M})$ and PPAR $\gamma$ siRNA under Th17-polarizing conditions for 4 days in the presence of madecassic acid $(3 \mu \mathrm{M})$. (a) Naive T cells were treated d with GW9662 $(1 \mu \mathrm{M})$ and T0070907 $(1 \mu \mathrm{M})$ under Th17-polarizing conditions for 4 days in the presence of madecassic acid $(3 \mu \mathrm{M})$. The proportions of CD4 ${ }^{+} \mathrm{IL}-17^{+} \mathrm{T}$ cells and $\mathrm{CD}^{+}{ }^{+} \mathrm{Fxp}^{+} \mathrm{T}$ cells were evaluated by flow cytometry. (b) Naive T cells were treated with madecassic acid $(3 \mu \mathrm{M})$ and PPAR $\gamma$ siRNA under Th17-polarizing conditions for 4 days. The proportions of $C D 4^{+} I L-17^{+} \mathrm{T}$ cells and $\mathrm{CD} 4^{+} \mathrm{Foxp}^{+} \mathrm{T}$ cells were evaluated by flow cytometry. (c) The relative expression of PPAR $\gamma$, ACC1 and Foxp3 was evaluated by immunofluorescence in the presence of madecassic acid $(3 \mu \mathrm{M})$, GW9662 $(1 \mu \mathrm{M})$ and T0070907 $(1 \mu \mathrm{M})$. (d) The relative expression of ACC1 and Foxp3 was evaluated by immunofluorescence in the presence of madecassic acid $(3 \mu \mathrm{M})$ and PPAR $\gamma$ siRNA. (e) Naive T cells were treated with GW9662 $(1 \mu \mathrm{M})$ and T0070907 (1 $\mu \mathrm{M})$ under Th17polarizing conditions for 4 days in the presence of madecassic acid $(3 \mu \mathrm{M})$. The relative expression of $p$-AMPK, ACC1 and Foxp3 was evaluated by western blot. (f) Naive T cells were treated with madecassic acid $(3 \mu \mathrm{M})$ and PPAR $\gamma$ siRNA under Th17-polarizing conditions for 4 days. The relative expression of p-AMPK, ACC1 and Foxp3 was evaluated by western blot. (g) Naive T cells were treated with madecassic acid $(3 \mu \mathrm{M})$ and AMPK siRNA under Th17-polarizing conditions for 4 days. The relative expression of cytosolic and nuclear PPAR $\gamma$ was measured by western blot. GAPDH was used as a cytoplasm marker; Lamin B1 was used as a nuclear marker. All data were expressed as means \pm S.E.M., $n=3 .{ }^{\#} P<0.05,{ }^{\# \#} P<0.01$ versus Th0 group; ${ }^{*} P<0.05,{ }^{* *} P<0.01$ versus Th17 group; ${ }^{\$} P<0.05,{ }^{\$ \$} P<0.01$ versus madecassic acid group 
a

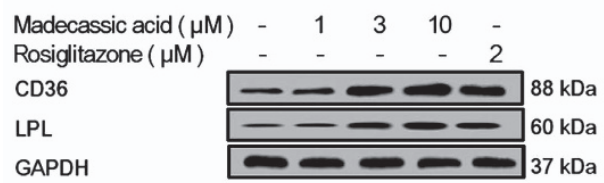

b

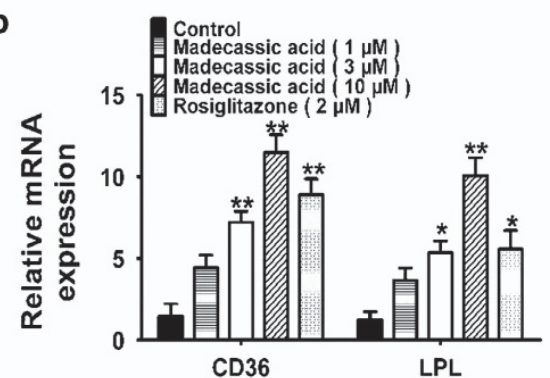

e

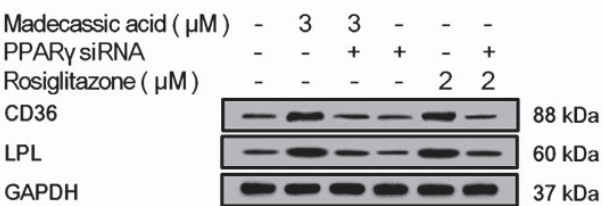

g

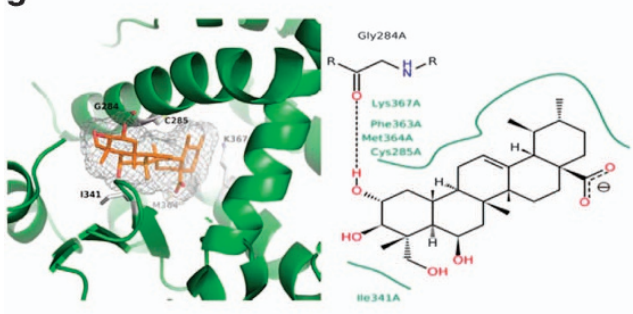

i

Madecassic acid $(\mu \mathrm{M})$ - $\quad \begin{array}{lllllll} & 3 & 3 & 3 & - & - & -\end{array}$

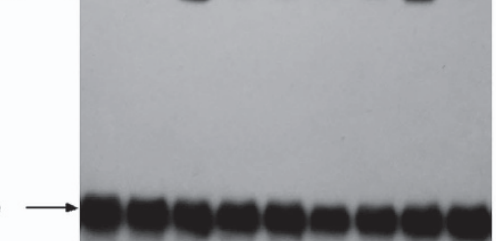

C

Madecassic acid $(\mu \mathrm{M}) \quad-\quad 3 \quad 3 \quad 3 \quad-$

$\begin{array}{llllllll}\mathrm{GW} 9662(\mu \mathrm{M}) & - & - & 1 & - & 1 & - & - \\ \mathrm{T} 0070907(\mu \mathrm{M}) & - & - & - & 1 & - & 1 & -\end{array}$

Rosiglitazone $(\mu \mathrm{M}) \quad-\quad-\quad-\quad-\quad-2$

CD36

LPL $\quad------160 \mathrm{kDa}$

GAPDH $37 \mathrm{kDa}$
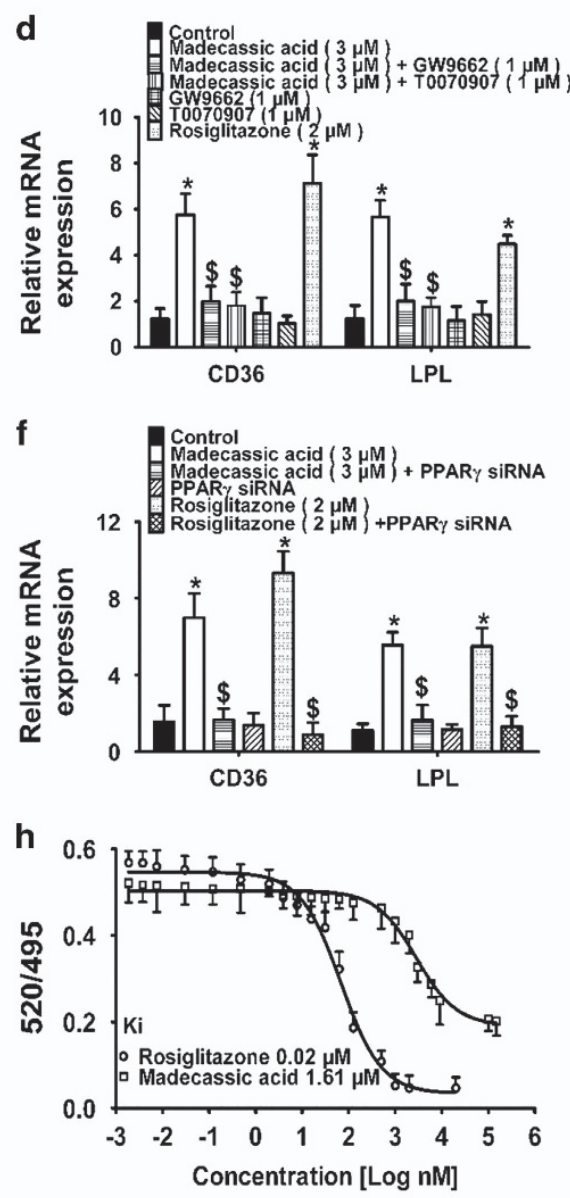

j

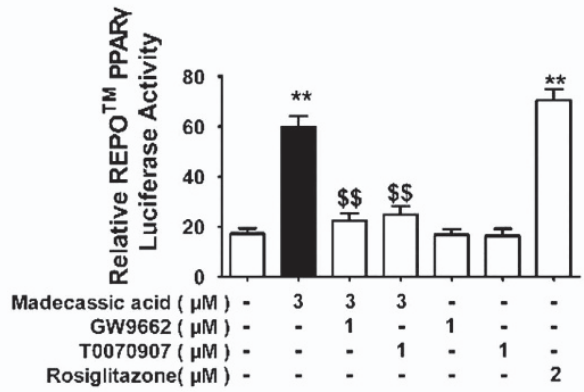

Figure 7 Madecassic acid as a PPAR $\gamma$ agonist. (a and $\mathbf{b})$ EL-4 cells were treated with madecassic acid $(1,3,10 \mu \mathrm{M})$ and rosiglitazone $(2 \mu \mathrm{M})$ for $24 \mathrm{~h}$. The expression of the PPAR $\gamma$-responsive genes CD36 and LPL was quantified by western blot and real-time PCR. (c and d) EL-4 cells were treated with madecassic acid ( $3 \mu \mathrm{M})$, GW9662 (1 $\mu \mathrm{M})$ and T0070907 $(1 \mu \mathrm{M})$ for $24 \mathrm{~h}$. The expression of CD36 and LPL was measured by western blot and real-time PCR. (e and f) EL-4 cells were treated with madecassic acid ( $3 \mu \mathrm{M})$ and PPAR $\gamma$ siRNA for $24 \mathrm{~h}$. The expression levels of CD36 and LPL were measured by western blot and real-time PCR. (g) The docking between madecassic acid and PPAR $\gamma$-LBD in EL-4 cells. (h) Binding of madecassic acid to PPAR $\gamma$-LBD in a competitive TR-FRET assay. (i) The effect of madecassic acid on the nuclear accumulation of PPAR $\gamma$ in EL-4 cells was determined by an EMSA in EL-4 cells. (j) The effect of madecassic acid on PPAR $\gamma$-dependent transactivation in EL-4 cells was assayed with a luciferase reporter gene assay. GAPDH was used as a cytoplasmic marker. The data were expressed as means \pm S.E.M., $n=3 .{ }^{\star} P<0.05,{ }^{\star \star} P<0.01$ versus control group, ${ }^{\$} P<0.05$, ${ }^{\$ \$} P<0.01$ versus madecassic acid group 


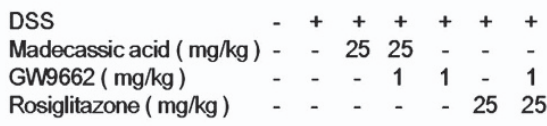

Rosiglitazone ( $\mathrm{mg} / \mathrm{kg}$ ) _ $\quad$ - $\quad$ - $\quad$ - $\quad$ - 2525

CD36

LPL

GAPDH

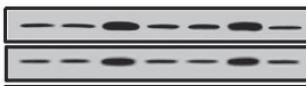

$-\infty-\infty-1$

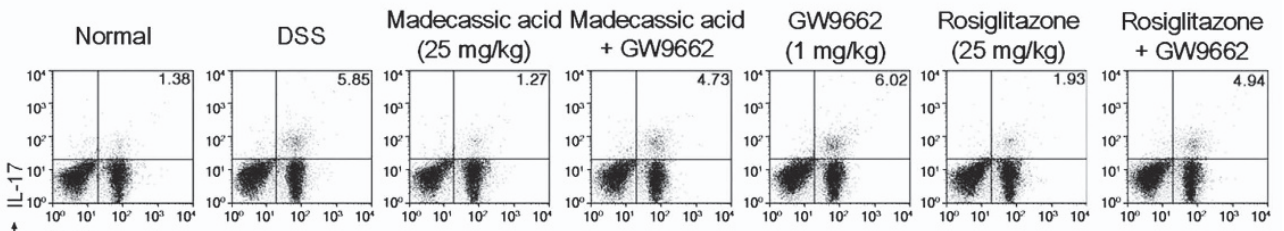

$\longrightarrow$ CD4
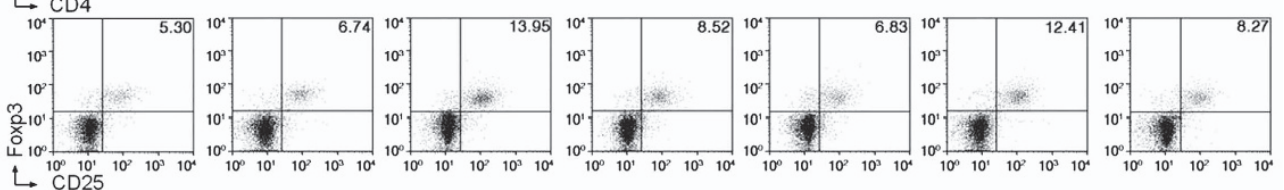

d

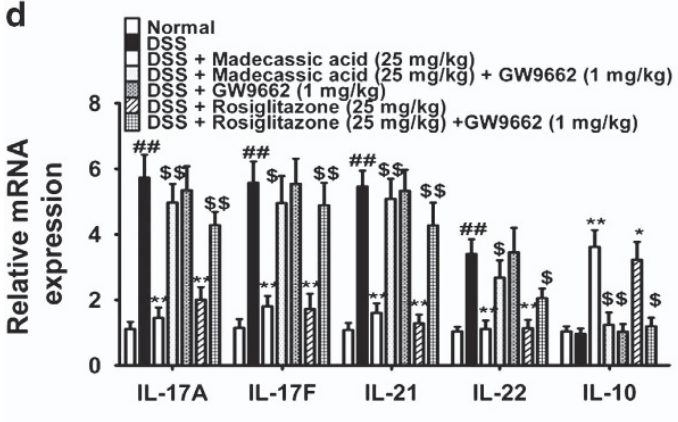

f

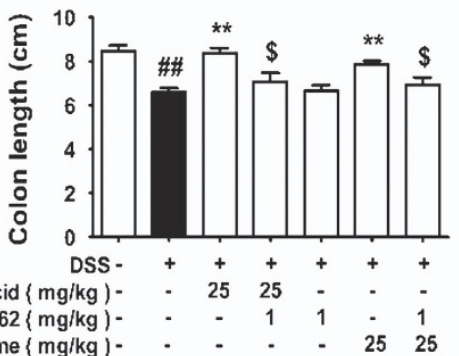

b DSS

$-++++++$

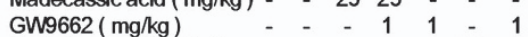

Rosiglitazone $(\mathrm{mg} / \mathrm{kg}) \quad-\quad-\quad-\quad-\quad 2525$

p-AMPK

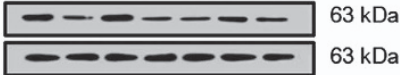

$---\infty-\infty \mathrm{kDa}$

$-\ldots-\ldots-\infty \mathrm{kDa}$

$-\infty-\infty \mathrm{kDa}$

Foxp3

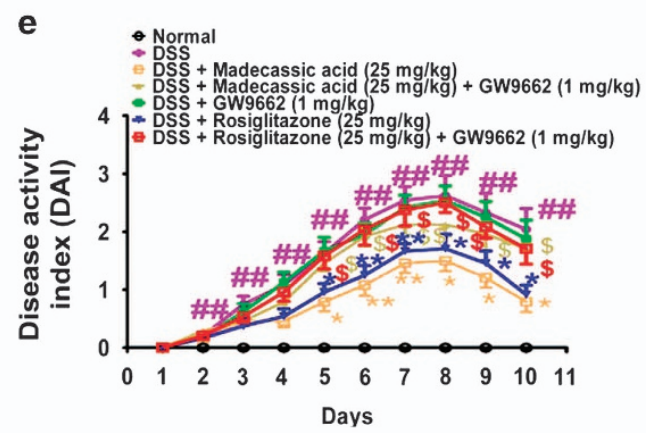

g

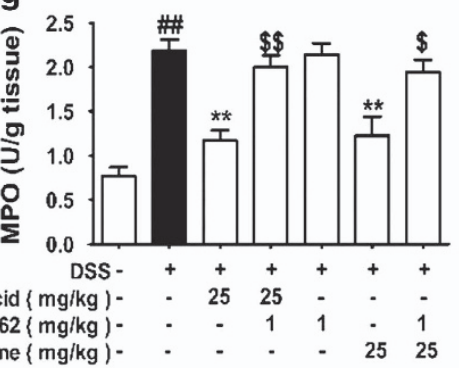

h
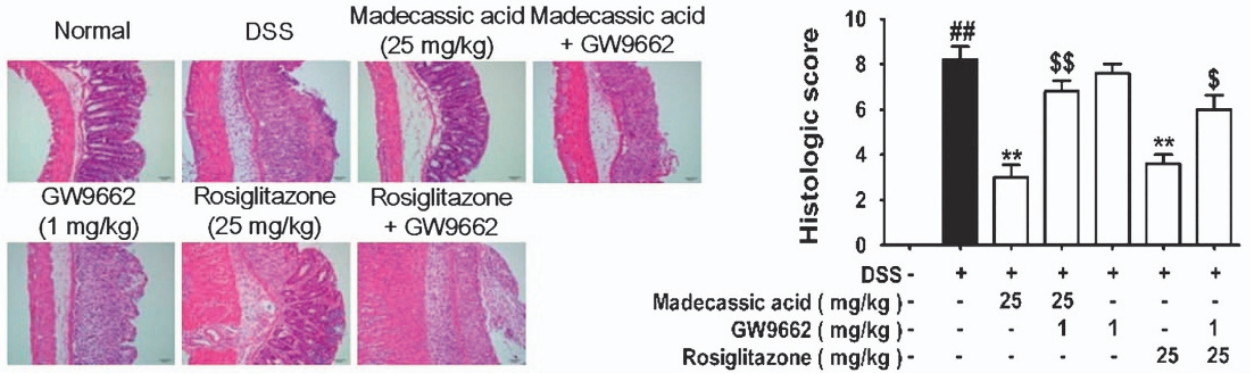

Figure 8 Madecassic acid recovered T helper type 17 (Th17)/regulatory T (Treg) balance in DSS-induced mice colitis through the PPAR $\gamma /$ AMPK/ACC1 pathway. Mice were fed 2.5\% DSS for 7 days and then normal water for 3 days. Madecassic acid (25 mg/kg) and rosiglitazone (25 mg/kg) were orally administered for 10 days. GW9662 (1 mg/kg) was administered by an intraperitoneal injection. (a) The expression of CD36 and LPL was analyzed by western blot. (b) The expression of p-AMPK, ACC1 and Foxp3 was detected by western blot. (c) The percentages of Th17 and Treg cells were measured by flow cytometry. (d) The levels of IL-17A, IL-17F, IL-21, IL-22 and IL-10 were assessed by real-time PCR. (e) DAl scores. (f) Colon length. (g) MPO activity. (h) H\&E scores. GAPDH was used as a cytoplasm marker. The data were expressed as means \pm S.E.M., $n=5$-8. ${ }^{\#} P<0.05,{ }^{\# \#} P<0.01$ versus normal group; ${ }^{*} P<0.05$, ${ }^{* *} P<0.01$ versus DSS group ${ }^{\$} P<0.05,{ }^{\$ \$} P<0.01$ versus madecassic acid group 
and incubated with various concentrations of madecassic acid $(1,3,10 \mu \mathrm{M})$, GW9662, T0070907 and rosiglitazone for $24 \mathrm{~h}$ at $37^{\circ} \mathrm{C}$ and $5 \% \mathrm{CO}_{2}$ and then analyzed by western blot, real-time PCR, immunofluorescence, EMSA or luciferase reporter gene assay.

Enzyme-linked immunosorbent assay. Colonic tissues isolated from DSS-treated mice were homogenates, and the supernatants were collected for the detection of IL-17A, IL-17F, IL-21, IL-22 and IL-10 level with assays by enzymelinked immunosorbent assay kits according to the manufacturer's instructions.

Intracellular staining and flow cytometry. Cells were harvested from in vitro culture or extracted from colonic lamina propria on day 10 after DSS induction for staining of intracellular IFN- $\gamma$, IL-4, IL-17A and Foxp3. For Th1, Th2 and Th17 intracellular staining, the cells were prestimulated with cell stimulation cocktail in $37^{\circ} \mathrm{C}$ for $5 \mathrm{~h}$ and then stained with FITC-anti-CD4 for $30 \mathrm{~min}$ followed by fixation and permeabilization and subsequently exposed to PE-anti-IFN- $\gamma$, APC-antiIL-17A and APC-anti-IL-4 antibodies for $1 \mathrm{~h}$. For Treg staining, the cells were cultured with FITC-conjugated-CD4 and PE-conjugated-CD25 for 30 min followed by fixation and permeabilization and subsequently stained with APC-conjugatedFoxp3 antibodies for $1 \mathrm{~h}$. All flow cytometric measurements were conducted on a FACS Calibur (BD Biosciences, San Jose, CA, USA). ${ }^{57,58}$

Gene expression analysis. The total RNA from colonic homogenates or cultured cells was extracted with Trizol reagent according to the manufacturer's instructions and then transcribed into cDNA using HiScript RT SuperMix. Then the expression of T-bet, GATA-3, RORyt, Foxp3, ACC1, CD36, LPL, IL-17A, IL-17F, IL-21, IL-22 and IL-10 were analyzed by Ace qPCR SYBR Green Master Mix with MyiQ2 Detection System. Gene expression was normalized to GAPDH and expressed relative to the normal group according to the $2^{-\Delta \Delta \mathrm{Ct}}$ method.

Western blot. Whole-cell lysates or proteins were prepared using the lysis buffer. The proteins were separated by $8 \%$ SDS-PAGE gel and further transferred to polyvinylidene fluoride (PVDF) membranes. After blocking with $5 \%$ nonfat milk for $2 \mathrm{~h}$, the PVDF membranes were incubated overnight at $4{ }^{\circ} \mathrm{C}$. Immunoblotting was performed using specific primary antibodies and incubated with goat anti-rabbit horseradish peroxidase for $2 \mathrm{~h}$ at $37^{\circ} \mathrm{C}$. Detection was visualized using the Odyssey Infrared Imaging System (LI-COR, Inc., Lincoln, NA, USA). ${ }^{57}$

Transient transfection. The EL-4 cells or purified $\mathrm{CD}^{+} \mathrm{T}$ cells were inoculated in six-well plates $\left(1 \times 10^{6}\right.$ cells/well). PPAR $\gamma$-specific siRNA (PPAR $\gamma$ siRNA), ACC1-specific siRNA (ACC1 siRNA), AMPK-specific siRNA (AMPK siRNA) or non-target-specific control siRNA (Control siRNA) plasmids were transiently transfected using Lipofectamine 2000 transfection reagent according to the manufacturer's instructions. After transfection, the cells were added with madecassic acid and cultured for 24 or $96 \mathrm{~h}$, respectively, and the proteins or total RNA were harvested for subsequent studies.

Molecular docking. The agonist-bound PPAR (Protein Data Bank: 4XLD) from the RCSB Protein Data Bank was chosen as the optimal template. The sequence alignment was adjusted according to the GPCR alignment constraints. The best model was generated, sorted by the GBVI/WSA dG score, energy minimized and hydrogens were added using Protonate3D in MOE. The final model was subjected to a binding site analysis and docking study. ${ }^{59}$

PPAR $y$ competitive binding assay. The LanthaScreen TR-FRET PPAR $\gamma$ competitive binding assay was applied according to the manufacturer's protocol. Madecassic acid or rosiglitazone was cultured with GST-fused human PPAR $\gamma$-LBD, terbium-labeled anti-GST antibody and a fluorescently labeled PPAR ligand for $3 \mathrm{~h}$ in the dark at room temperature. The FRET signal was valued by excitation at $340 \mathrm{~nm}$ and emission at $520 \mathrm{~nm}$ for fluorescein and $495 \mathrm{~nm}$ for terbium. The ability of binding to the PPAR $\gamma$-LBD was measured by the downregulation of the $520 \mathrm{~nm} /$ $495 \mathrm{~nm}$ ratio. ${ }^{59,60}$

Electrophoretic mobility shift assay. The DNA-binding activity of PPAR $\gamma$ was measured by EMSA using a commercial kit. In brief, biotin-labeled PPAR $\gamma$-specific oligonucleotides were synthesized as the labeled probe according to the manufacturer's instructions. Nuclear extracts were incubated with poly (dl$\mathrm{dC}$ ), labeled probe and binding buffer at $25^{\circ} \mathrm{C}$ for $10 \mathrm{~min}$. The reaction mixtures were then separated with $5 \%$ non-denatured polyacrylamide gels at $1 \mathrm{~mA} / \mathrm{cm}$ at
$4{ }^{\circ} \mathrm{C}$ for $1.5 \mathrm{~h}$ and transferred to a PVDF membrane. The biotin end-labeled DNA was tested with a streptavidin-HRP conjugate and a chemiluminescent substrate. The membrane was finally detected with X-ray film and analyzed with the Quantity One software (BD Biosciences, San Jose, CA, USA). ${ }^{61}$

Luciferase reporter assay. EL-4 cells were incubated in 96-well plates $\left(1 \times 10^{4} \mathrm{cell} / \mathrm{s} / \mathrm{ml}\right)$, and the cells in each well were co-transfected with PPRE-REPO. A PPRE-driven luciferase reporter plasmid was applied for examining specific activation of PPAR $\gamma$ binding to the PPRE. The cells were suspended in fresh culture medium and exposed to madecassic acid in the presence of GW9662 and T0070907, either alone or in a combination for $12 \mathrm{~h}$. Then the cells were lysed, and the supernatants were collected. The luciferase activity was measured by a luciferase assay system and a multimode reader. ${ }^{62}$

Statistical analysis. The data were presented as the means \pm S.E.M. from at least three independent experiments. Statistical analysis was performed by using one-way analysis of variance followed by Dunnett's test. $P<0.05$ were accepted as a significant difference.

\section{Conflict of Interest}

The authors declare no conflict of interest.

Acknowledgements. This work was supported by National Natural Science Foundation of China (No. 81374038), the Scientific Innovation Research of College Graduates in Jiangsu Province (No. KYLX16_1207) and the Priority Academic Program Development of Jiangsu Higher Education Institutions.

\section{Author contributions}

$\mathrm{XX}$ and $\mathrm{YW}$ designed and performed experiments. PZ analyzed the data. WW assisted partial experiments. $B T$ and $Z W$ provided helpful suggestions. $Y D$ and $Y X$ guided researches and revised manuscript.

1. Conrad K, Roggenbuck D, Laass MW. Diagnosis and classification of ulcerative colitis. Autoimmun Rev 2014; 13: 463-466.

2. Ananthakrishnan AN. Epidemiology and risk factors for IBD. Nat Rev Gastroenterol Hepatol 2015; 12: 205-217.

3. De Souza HS, Fiocchi C. Immunopathogenesis of IBD: current state of the art. Nat Rev Gastroenterol Hepatol 2016; 13: 13-27.

4. Eastaff-Leung N, Mabarrack N, Barbour A, Cummins A, Barry S. Foxp3+ regulatory T cells, Th17 effector cells, and cytokine environment in inflammatory bowel disease. J Clin Immunol 2010; 30: 80-89.

5. Maloy KJ, Powrie F. Intestinal homeostasis and its breakdown in inflammatory bowel disease. Nature 2011; 474: 298-306.

6. Geremia A, Biancheri P, Allan P, Corazza GR, Di Sabatino A. Innate and adaptive immunity in inflammatory bowel disease. Autoimmun Rev 2014; 13: 3-10.

7. Abraham C, Cho JH. IL-23 and autoimmunity: new insights into the pathogenesis of inflammatory bowel disease. Annu Rev Med 2009; 60: 97-110.

8. Neurath MF. Cytokines in inflammatory bowel disease. Nat Rev Immunol 2014; 14: 329-342.

9. Chandrika UG, Prasad Kumarab PA. Gotu kola (Centella asiatica): nutritional properties and plausible health benefits. Adv Food Nutr Res 2015; 76: 125-157.

10. James JT, Dubery IA. Pentacyclic triterpenoids from the medicinal herb, Centella asiatica (L.) Urban. Molecules 2009; 14: 3922-3941.

11. Azerad R. Chemical structures, production and enzymatic transformations of sapogenins and saponins from Centella asiatica (L.) Urban. Fitoterapia 2016; 114: 168-187.

12. Wang T, Wei ZF, Dou YN, Yang Y, Leng DD, Kong LY et al. Intestinal interleukin-10 mobilization as a contributor to the anti-arthritis effect of orally administered madecassoside: a unique action mode of saponin compounds with poor bioavailability. Biochem Pharmacol 2015; 94: 30-38.

13. Weaver CT, Hatton RD. Interplay between the Th17 and Treg cell lineages: a (co-) evolutionary perspective. Nat Rev Immunol 2009; 9: 883-889.

14. Gagliani N, Amezcua Vesely MC, Iseppon A, Brockmann L, Xu H, Palm NW et al. Th17 cells trans-differentiate into regulatory $T$ cells during resolution of inflammation. Nature 2015; 523 : 221-225.

15. Park BV, Pan F. The role of nuclear receptors in regulation of Th17/Treg biology and its implications for diseases. Cell Mol Immunol 2015; 12: 533-542.

16. Sekiya T, Kashiwagi I, Inoue N, Morita R, Hori S, Waldmann $\mathrm{H}$ et al. The nuclear orphan receptor nr4a2 induces Foxp3 and regulates differentiation of CD4+ T cells. Nat Commun 2011; 2: 269.

17. Daynes RA, Jones DC. Emerging roles of PPARs in inflammation and immunity. Nat Rev Immunol 2002; 2: 748-759. 
18. Shan M, You R, Yuan X, Frazier MV, Porter P, Seryshev A et al. Agonistic induction of PPAR $\gamma$ reverses cigarette smoke-induced emphysema. J Clin Invest 2014; 124: 1371-1381.

19. Cipolletta D, Feuerer M, Li A, Kamei N, Lee J, Shoelson SE et al. PPAR $\gamma$ is a major driver of the accumulation and phenotype of adipose tissue Treg cells. Nature 2012; 486: 549-553.

20. Poliska S, Penyige A, Lakatos PL, Hungarian IBD Study Group, Papp M, Palatka K et al. Association of PPAR $\gamma$ polymorphisms with inflammatory bowel disease in a hungarian cohort. Inflamm Bowel Dis 2012; 18: 472-479.

21. Peyrin-Biroulet L, Beisner J, Wang G, Nuding S, Oommen ST, Kelly D et al. Peroxisome proliferator-activated receptor $\gamma$ activation is required for maintenance of innate antimicrobial immunity in the colon. Proc Natl Acad Sci USA 2010; 107: 8772-8777.

22. Bian DF, Zhang JZ, Wu X, Dou YN, Yang Y, Tan $Q$ et al. Asiatic acid isolated from Centella asiatica inhibits TGF- $\beta 1$ induced collagen expression in human keloid fibroblasts via PPAR $\gamma$ activation. Int J Biol Sci 2013; 9: 1032-1042.

23. Castellano JM, Guinda A, Delgado T, Rada M, Cayuela JA. Biochemical basis of the antidiabetic activity of oleanolic acid and related pentacyclic triterpenes. Diabetes 2013; 62 : 1791-1799.

24. Bramhall M, Flórez-Vargas O, Stevens R, Brass A, Cruickshank S. Quality of methods reporting in animal models of colitis. Inflamm Bowel Dis 2015; 21: 1248-1259.

25. Hirota K, Martin B, Veldhoen M. Development, regulation and functional capacities of Th17 cells. Semin Immunopathol 2010; 32: 3-16.

26. Lee YK, Mukasa R, Hatton RD, Weaver CT. Developmental plasticity of Th17 and Treg cells Curr Opin Immunol 2009; 21: 274-280.

27. Berod L, Friedrich C, Nandan A, Freitag J, Hagemann S, Harmrolfs K et al. De novo fatty acid synthesis controls the fate between regulatory T and T helper 17 cells. Nat Med 2014; 20: 1327-1333.

28. Endo Y, Asou HK, Matsugae N, Hirahara K, Shinoda K, Tumes DJ et al. Obesity drives Th17 cell differentiation by inducing the lipid metabolic kinase, ACC1. Cell Rep 2015; 12 1042-1055

29. O'Neill LA, Hardie DG. Metabolism of inflammation limited by AMPK and pseudo-starvation. Nature 2013; 493: 346-355.

30. Jeon SM. Regulation and function of AMPK in physiology and diseases. Exp Mol Med 2016; 48: e245.

31. Saleh M, Elson CO. Experimental inflammatory bowel disease: insights into the host-microbiota dialogue. Immunity 2011; 34: 293-302

32. Oukka M. Interplay between pathogenic Th17 and regulatory T cells. Ann Rheum Dis 2007; 66: 87-90

33. O'Connor W Jr, Zenewicz LA, Flavell RA. The dual nature of Th17 cells: shifting the focus to function. Nat Immunol 2010; 11: 471-476.

34. Zhang HL, Zheng YJ, Pan YD, Xie C, Sun H, Zhang YH et al. Regulatory T-cell depletion in the gut caused by integrin $\beta 7$ deficiency exacerbates DSS colitis by evoking aberrant innate immunity. Nat Rev Immunol 2015; 7: 875-888.

35. Noack M, Miossec $P$. Th17 and regulatory $T$ cell balance in autoimmune and inflammatory diseases. Autoimmun Rev 2014; 13: 668-677.

36. Omenetti S, Pizarro TT. The Treg/Th17 axis: a dynamic balance regulated by the gut microbiome. Front Immunol 2015; 6: 639.

37. Kong N, Lan Q, Chen M, Wang J, Shi W, Horwitz DA et al. Antigen-specific transforming growth factor $\beta$-induced Treg cells, but not natural Treg cells, ameliorate autoimmune arthritis in mice by shifting the Th17/Treg cell balance from Th17 predominance to Treg cell predominance. Arthritis Rheum 2012; 64: 2548-2558.

38. Nguyen TL, Sullivan NL, Ebel M, Teague RM, DiPaolo RJ et al. Antigen-specific TGF- $\beta$ induced regulatory $T$ cells but not natural Tregs ameliorate autoimmune arthritis by shifting the balance of Th17 toward Treg cells. J Immunol 2011; 187: 1745-1753.

39. Chang $\mathrm{CH}$, Pearce EL. Emerging concepts of $\mathrm{T}$ cell metabolism as a target of immunotherapy. Nat Immunol 2016; 17: 364-368.

40. Barbi J, Pardoll D, Pan F. Metabolic control of the Treg/Th17 axis. Immunol Rev 2013; 252: $52-77$

41. O'Sullivan D, Pearce EL. Targeting T cell metabolism for therapy. Trends Immunol 2015; 36 : 71-80.

42. Davis FP, Kanno Y, O'Shea JJ. A metabolic switch for Th17 pathogenicity. Cell 2015; 163 1308-1310.

43. Wang R, Green DR. Metabolic checkpoints in activated T cells. Nat Immunol 2012; 13: 907-915.

44. Hardie DG, Ross FA, Hawley SA. AMPK: a nutrient and energy sensor that maintains energy homeostasis. Nat Rev Mol Cell Biol 2012; 13: 251-262.

45. Vincent EE, Coelho PP, Blagih J, Griss T, Viollet B, Jones RG. Differential effects of AMPK agonists on cell growth and metabolism. Oncogene 2015; 34: 3627-3639.
46. Park MJ, Lee SY, Moon SJ, Son HJ, Lee SH, Kim EK et al. Metformin attenuates graftversus-host disease via restricting mammalian target of rapamycin/signal transducer and activator of transcription 3 and promoting adenosine monophosphate-activated protein kinase-autophagy for the balance between T helper 17 and Tregs. Transl Res 2016; 173 115-130.

47. Wang YX. PPARs: diverse regulators in energy metabolism and metabolic diseases. Cell Res 2010; 20: 124-137.

48. Lempradl A, Pospisilik JA, Penninger JM. Exploring the emerging complexity in transcriptional regulation of energy homeostasis. Nat Rev Genet 2015; 16: 665-681.

49. Glass CK, Saijo K. Nuclear receptor trans-repression pathways that regulate inflammation in macrophages and T cells. Nat Rev Immunol 2010; 10: 365-376.

50. Pedersen G, Brynskov J. Topical rosiglitazone treatment improves ulcerative colitis by restoring peroxisome proliferator-activated receptor $\gamma$ activity. Am J Gastroenterol 2010; 105: 1595-1603.

51. Hontecillas R, Bassaganya-Riera J. Peroxisome proliferator-activated receptor $\gamma$ is required for regulatory CD4+ T cell-mediated protection against colitis. J Immunol 2007; 178 : 2940-2949.

52. Hontecillas R, Horne WT, Climent M, Guri AJ, Evans C, Zhang Y et al. Immunoregulatory mechanisms of macrophage PPAR $\gamma$ in mice with experimental inflammatory bowel disease. Mucosal Immunol 2011; 4: 304-313.

53. Adachi M, Kurotani R, Morimura K, Shah Y, Sanford M, Madison BB et al. Peroxisome proliferator activated receptor $\gamma$ in colonic epithelial cells protects against experimental inflammatory bowel disease. Gut 2006; 55: 1104-1113.

54. Hontecillas R, Roberts PC, Carbo A, Vives C, Horne WT, Genis S et al. Dietary abscisic acid ameliorates influenza virus-associated disease and pulmonary immunopathology through a PPAR $\gamma$-dependent mechanism. J Nutr Biochem 2013; 24: 1019-1027.

55. Sánchez-Hidalgo M, Martín AR, Villegas I, Alarcón De La Lastra C. Rosiglitazone, an agonis of peroxisome proliferator-activated receptor gamma, reduces chronic colonic inflammation in rats. Biochem Pharmacol 2005; 69: 1733-1744.

56. Wirtz S, Neufert C, Weigmann B, Neurath MF. Chemically induced mouse models of intestinal inflammation. Nat Protoc 2007; 2: 541-546.

57. Wu X, Dou YN, Yang Y, Bian DF, Luo JQ, Tong B et al. Arctigenin exerts anti-colitis efficacy through inhibiting the differentiation of Th1 and Th17 cells via an mTORC1-dependent pathway. Biochem Pharmacol 2015; 196: 323-336.

58. Ueno A, Jijon H, Chan R, Ford K, Hirota C, Kaplan GG. Increased prevalence of circulating novel IL-17 secreting Foxp3 expressing CD4+ T cells and defective suppressive function of circulating Foxp3+ regulatory cells support plasticity between Th17 and regulatory T cells in inflammatory bowel disease patients. Inflamm Bowel Dis 2013; 19: 2522-2534.

59. Weidner C, de Groot JC, Prasad A, Freiwald A, Quedenau C, Kliem M et al. Amorfrutins are potent antidiabetic dietary natural products. Proc Natl Acad Sci USA 2012; 109: 7257-7262.

60. Nevin DK, Peters MB, Carta G, Fayne D, Lloyd DG et al. Integrated virtual screening for the identification of novel and selective peroxisome proliferator-activated receptor (PPAR) scaffolds. J Med Chem 2012; 55: 4978-4989.

61. Lee YJ, Ko EH, Kim JE, Kim E, Lee H, Choi $\mathrm{H}$ et al. Nuclear receptor PPAR $\gamma$ regulated monoacylglycerol 0-acyltransferase1 (MGAT1) expression is responsible for the lipid accumulation in diet-induced hepatic steatosis. Proc Natl Acad Sci USA 2012; 109 13656-13661.

62. Knape T, Flesch D, Kuchler L, Sha LK, Giegerich AK, Labocha S et al. Identification and characterisation of a prototype for a new class of competitive PPAR $\gamma$ antagonists. Eur $J$ Pharmacol 2015; 755: 16-26.

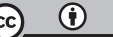

Cell Death and Disease is an open-access journal published by Nature Publishing Group. This work is licensed under a Creative Commons Attribution 4.0 International License. The images or other third party material in this article are included in the article's Creative Commons license, unless indicated otherwise in the credit line; if the material is not included under the Creative Commons license, users will need to obtain permission from the license holder to reproduce the material. To view a copy of this license, visit http://creativecommons.org/licenses/by/4.0/

(C) The Author(s) 2017 